\begin{tabular}{|c|c|c|}
\hline Beitr. Ent. & Berlin & ISSN 0005-805X \\
\hline $\mathbf{5 0}(2000) 2$ & S. $487-518$ & 02.10 .2000 \\
\hline
\end{tabular}

\title{
Vergleichende ökologische Untersuchungen an Epitheca bima- culata CHARPENTIER 1825 im Saarland und in der Uckermark
}

\section{(Odonata: Corduliidae)}

Mit 13 Tabellen und 11 Figuren

BERND TROCKUR und RÜDIGER MAUERSBERGER ${ }^{1}$

\section{Zusammenfassung}

Zwischen 1988 und 1998 wurde diese als sehr selten geltende Art im Saarland (Südwestdeutschland) und in der Uckermark (Nordostdeutschland) an über 100 Gewässern entdeckt (TROCKUR \& MAUERSBERGER in prep.). Ein großer Teil der Habitate der Art in diesen beiden Regionen wurde in Bezug auf zahlreiche Parameter untersucht und verglichen.

Im Saarland besiedelt die Art eutrophe bis polytrophe künstliche Altwässer, Kiesgruben und Fischteiche in der Auenlandschaft. In der Uckermark handelt es sich bei den meisten Fundorten um eutrophe, natürliche Kleinseen vorwiegend in Endmoränennähe.

Als hervorstechende Merkmale der Epitheca-Gewässer in beiden Gebieten dürfen gelten:

- stehende Gewässer geringer Größe: $85 \%$ der Fundgewässer sind kleiner als 10 ha, $63 \%$ unter 4 ha;

- relativ flache, zumeist ungeschichtete Wasserkörper: Maximaltiefe zwischen 1,1 und $12 \mathrm{~m}(91 \%$ unter $8 \mathrm{~m}, 67 \%$ zwischen 2 und $6 \mathrm{~m}$ ), nur $12 \%$ mit vollständiger Temperaturschichtung;

- sommerliches Sauerstoffdefizit in der Tiefe: nur wenige Gewässer besitzen noch über $2 \mathrm{mg} / 1 \mathrm{O}_{2}$ unterhalb 4 m Tiefe;

- von Phytoplankton getrübte, eutrophe bis polytrophe Gewässer: Fortpflanzungsnachweise gelangen an Gewässern mit mittleren Sichttiefen zwischen 0,5 und 3,1 m (68\% mit Sichttiefen unter $2 \mathrm{~m})$;

- Strukturen für Männchenreviere und als Eiablagesubstrat: ausgedehnte Schwimmblatt- oder oberflächennahe Submersvegetation (an $80 \%$ der Gewässer), seltener auch umgestürzte Bäume oder Röhrichthalme;

- alle Gewässer sind von Ufergehölzen und/oder von Wald umgeben, in dem Laubbäume dominieren;

- alle Gewässer weisen Fischbestände auf;

- die Libellengemeinschaften werden von Orthetrum cancellatum, Cordulia aenea und ErythrommaArten dominiert; die Besiedlungsdichte von Aeshniden ist zumeist gering.

Bisher mehrfach in der Literatur als charakteristisch genannte Gewässertypen können somit nicht als die wichtigen Epitheca-Habitate angesehen werden: große Klarwasserseen oder schwach saure Moorweiher. Als typischen Primärlebensraum der Art in Europa sehen wir Auengewässer und Kleinseen an, in denen ein nicht zu geringer Fischbestand die Biozönose steuert.

'gewidmet Dr. GOTTFRIED MAUERSBERGER (7.10.1931-17.05.1994).

Bei einer gemeinsamen Exkursion zum Brackensee am 14.05.94 konnte ich ihm einen lang gehegten Wunsch erfüllen: wir betrachteten ein Epitheca-Exemplar aus nächster Nähe, kurz nach dem Schlupf. Es war die letzte Libellenbeobachtung meines Vaters nach knapp 30 Jahren Beschäftigung mit dieser Tiergruppe. Drei Tage später starb er überraschend an einem Herzinfarkt. 


\section{Summary}

Between 1988 and 1998, more than a hundred habitats of the supposedly rare corduliid dragonfly Epitheca bimaculata were studied in the western parts of the Saarland (SW-Germany), in the adjacent parts of Luxembourg and France (especially the valleys of the rivers Saar and Mosel) and in the Uckermark (,Biosphärenreservat Schorfheide-Chorin“, NE- Germany). In the Saarland, the species inhabits eutrophic to polytrophic artificial backwaters, gravelpits and fish-ponds, whereas in the Uckermark it occurs in small natural, predominantly eutrophic lakes.

Relevant characteristics of the Epitheca-habitats in both regions were the following:

- still waters of small area: $85 \%$ of the habitats were less than 10 ha, $63 \%$ less than 4 ha;

- relatively shallow water body without distinct stratification: maximum water depth between 1.1 and $12 \mathrm{~m}(91 \%$ less than $8 \mathrm{~m}, 67 \%$ between 2 and $6 \mathrm{~m}$, only $12 \%$ with complete temperature stratification;

- low oxygen content in deeper strata: usually less than $2 \mathrm{mg} \mathrm{O}_{2} / 1$ below $4 \mathrm{~m}$;

- water clouded by phytoplankton, eutrophic to polytrophic: breeding sites between 0.5 and $3.1 \mathrm{~m}$ median Secchi-depth ( $68 \%$ with visibility of less than $2 \mathrm{~m}$ );

- water surface with abundant floating or submerged vegetation, more rarely also tree trunks or reed: substrates for oviposition and habitats of the younger larvae;

- water surrounded by shrubs and/or deciduous woodland;

- water inhabited by fish populations;

- the dragonfly communities are dominated by Orthetrum cancellatum, Cordulia aenea and Erythromma species; aeshnids usually occur a low densities.

The typical habitats of Epitheca in central and western Europe are not large, oligotrophic lakes or slightly acetous bog ponds, but still waters in the flood plain (river lagoons or gravel pits) and small lakes inhabited by fish populations.

\section{Keywords}

Corduliidae, limnology, Uckermark, Saarland Germany, co-existence, fish, habitat structure, broadleavered tree, forest, water vegetation, dragonfly community, lake, flood plain, optimal habitat, primary habitat, threat, negative factor.

\section{Inhaltsverzeichnis}

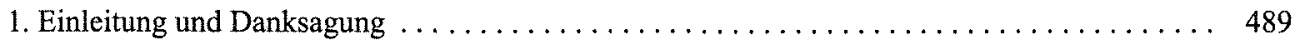

2. Untersuchungsgebiete $\ldots \ldots \ldots \ldots \ldots \ldots \ldots \ldots \ldots \ldots \ldots \ldots \ldots \ldots \ldots \ldots \ldots \ldots$

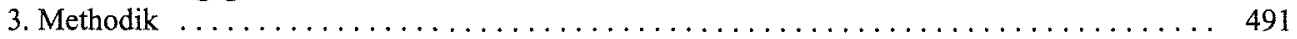

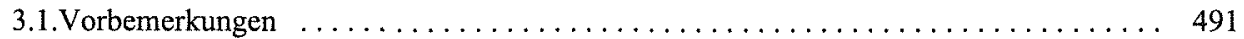

3.2. Faunistische Untersuchungen: Libellen und Fische . . . . . . . . . . . . 491

3.3. Limnologische Untersuchungen: Morphometrie, Schichtung, Wasserbeschaffenheit . . 492

3.4. Erfassung von Habitatstrukturen: Gewässervegetation, Sedimente, Umfeld . . . . . . . 492

3.5. Klassifikation des Status der Gewässer für Epitheca bimaculata . . . . . . . . . . . . 493

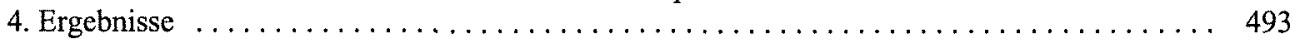

4.1. Gewässertyp, Hydrologie und Genese . . . . . . . . . . . . . . . . 493

4.2. Gewässerphysiognomie/Morphometrie . . . . . . . . . . . . . . . . . . 494

4.3. Temperatur-und Sauerstoffschichtung des Wasserkörpers $\ldots \ldots \ldots \ldots \ldots \ldots \ldots . \ldots 46$

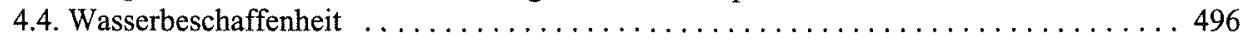

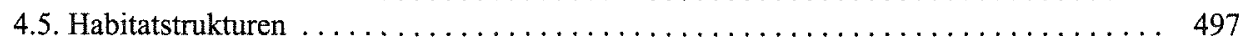

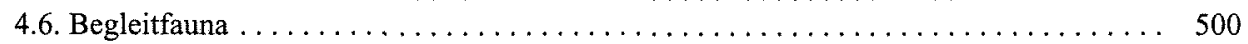

4.7. Zusammenfassende Darstellung der Ergebnisse $\ldots \ldots \ldots \ldots \ldots \ldots \ldots \ldots \ldots \ldots \ldots$

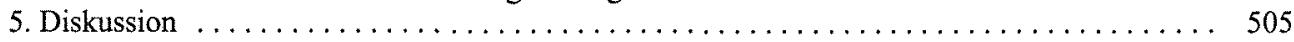

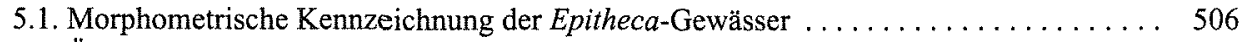

5.2. Über die geringe Bedeutung der Wasserbeschaffenheit für Epitheca . . . . . . . . . 507

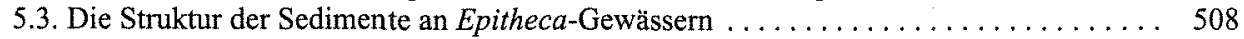


5.4. Bedeutung der Wasservegetation $\ldots \ldots \ldots \ldots \ldots \ldots \ldots \ldots \ldots \ldots \ldots \ldots \ldots \ldots$

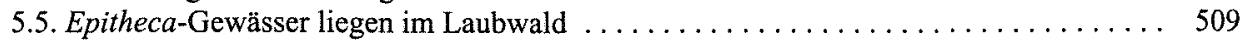

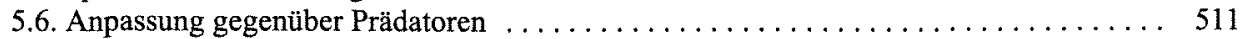

5.7. Bemerkungen zu Negativfaktoren, Optimalhabitat und typischem Landschaftsraum . . 512

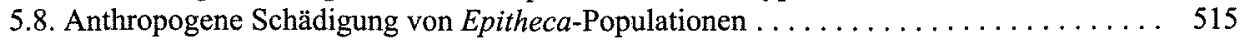

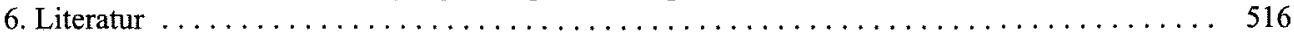

\section{Einleitung und Danksagung}

Epitheca bimaculata wurde in den letzten Jahren in Deutschland und auch in Frankreich vermehrt nachgewiesen. Erst seit kurzem bekannt sind die zahlreichen Vorkommen im Mittleren Saartal (TROCKUR, 1993) und in der brandenburgischen Uckermark nordöstlich von Berlin (MAUERSBERGer \& MAUERSBERger, 1992, 1996, TrockUr \& MAUERSBERGER, in prep.).

Die Kenntnisse über das Habitat sind relativ rar, zum Teil widersprüchlich oder so allgemein, daß nicht verständlich wird, weshalb die Art so selten ist (ROBERT, 1959, SCHORR, 1990). Wir betrachten in dieser Arbeit die beiden wichtigsten derzeit bekannten Kerngebiete der Verbreitung von Epitheca in Deutschland, die weit entfernt und zum jetzigen Stand der Erkenntnisse anscheinend auch voneinander separiert sind: Nachweise aus Sachsen, Thüringen, Hessen und Nordrhein-Westfalen stehen seit Jahrzehnten oder gar gänzlich aus. Schwerpunkt unserer Darlegungen ist die Analyse und der ökologische Vergleich von meist erst wenige Jahre bestehenden, künstlichen Gewässern im Saarland ( $\mathrm{n}=19)$ mit natürlich entstandenen Seen in der Uckermark $(n=49)$.

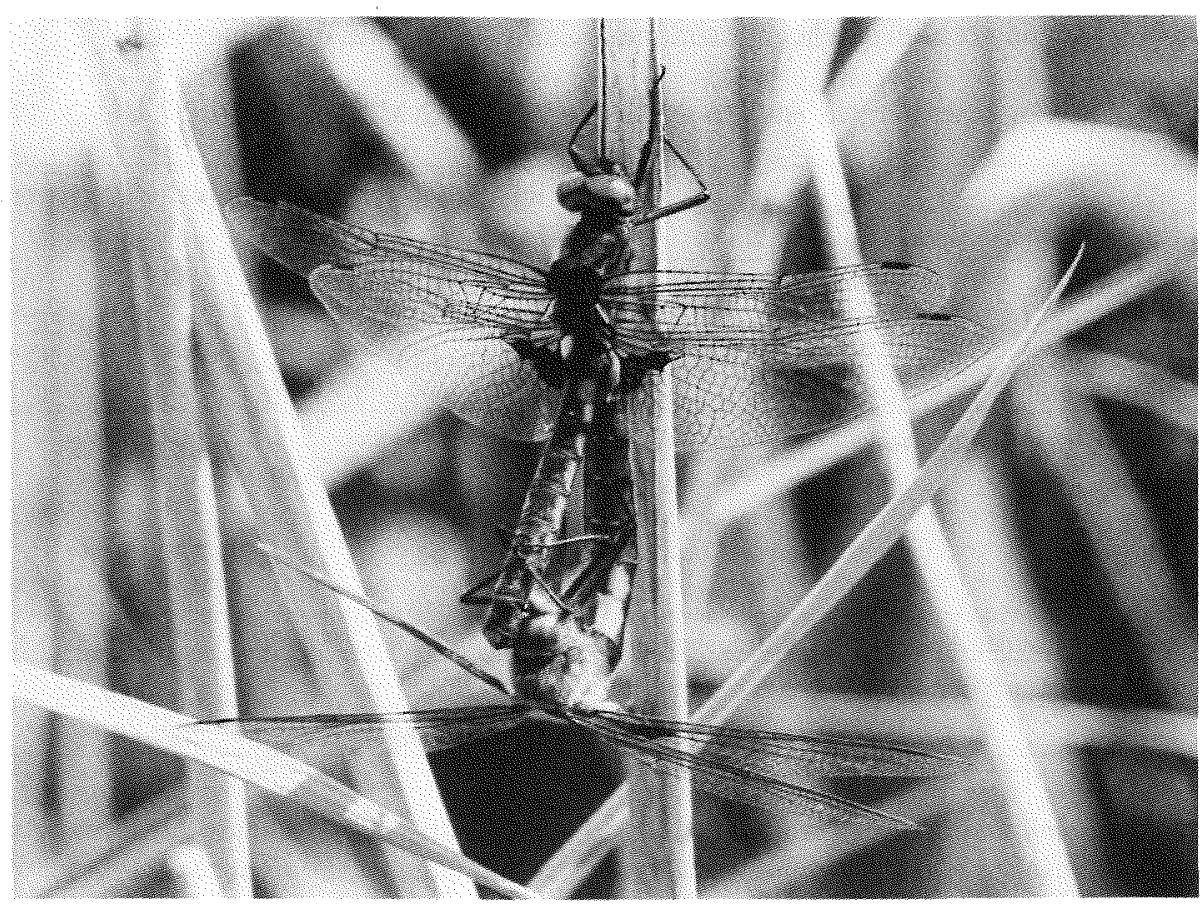

Fig. 1: Kopula der Zweiflecklibelle Epitheca bimaculata (Oberer Saaraltarm Beckingen, 24.5.1992). 


\section{Danksagung}

Für Informationen, Literaturhinweise, Diskussionen oder sonstige Unterstützung bedanken wir uns recht herzlich bei: Dr. AXEL DIDION (Homburg), MLADEN KOTARAC (Maribor, Slowenien) und MATJAC BEDJANIC (Fram, Slowenien), GENNARO COPPA (Flize, Frankreich), Dr. MARTIN SCHORR (Zerf), Dr. RAINER BUCHWALD (Freiburg), Dr. KLAUS STERNBERG (Stutensee), MATTHIAS KITT (Minfeld), AlFoNS EWEN (Beckingen), MATHIAS WEITZEL (Trier), Uli HEINTZ (Eppelborn), ROLF PESCHEL (Hamburg) und dem Büro für Landschaftsökologie (Nohfelden).

Besonderen Dank schulden wir Herrn Fischereimeister EIKE KÄHLER (Blankenburg), von dem mehrere der Exuvienaufsammlungen stammen.

Für die Genehmigung der Untersuchungen danken wir den jeweils zuständigen Naturschutzbehörden (Umweltminister des Saarlandes -Oberste Naturschutzbehörde- und Landesumweltamt Brandenburg).

\section{Untersuchungsgebiete}

Das Saarland liegt im Südwesten Deutschlands und grenzt an Frankreich und Luxemburg. Das Untersuchungsgebiet erstreckt sich überwiegend auf den nordwestlichen Bereich in Tallagen zwischen 140-305 m ü NN. Die Mehrzahl der Funde sowie alle Gewässer mit höheren Abundanzen liegen in der Rehlinger-Dillinger Talweitung des Mittleren Saartales, nur wenige Kilometer voneinander getrennt. Hier ist in den letzten Jahren/Jahrzehnten durch Saarausbau und Kiesgewinnung eine gewässerreiche Landschaft im sonst an natürlichen Stillgewässern armen, durch Gewerbe, Industrie und Wohnsiedlungen stark überprägten, relativ waldreichen Saarland entstanden. Im kleinsten Flächenland der Bundesrepublik leben etwa 420 Einwohner pro $\mathrm{km}^{2}$.

Die tiefer gelegenen Teile des Saarlandes, v.a. Mosel- und Saartal gelten ähnlich wie die Oberrheingegend als klimatische Gunsträume. Kleinflächig wird Wein angebaut.

Tab. 1: Klimatologischer Vergleich der Bearbeitungsgebiete (nach MEYNEN u. a., 1959, HEYER, 1962).

\begin{tabular}{|l|c|c|}
\hline & mittleres Saartal & südliche Uckermark \\
\hline Typisierung & subatlantisch & subkontinental \\
\hline Temperaturen & & \\
Januarmittel & $0,2-1,0^{\circ} \mathrm{C}$ & $-1,0^{\circ} \mathrm{C}$ \\
Julimittel & $17,1-18,0^{\circ} \mathrm{C}$ & $17,5-18,5^{\circ} \mathrm{C}$ \\
Jahresmittel & $8,7-9,0^{\circ} \mathrm{C}$ & $8,0-8,5^{\circ} \mathrm{C}$ \\
\hline Länge der Vegetationsperiode & 205 Tage & 225 Tage \\
\hline mittlere Anzahl der & & 25 \\
Eistage & 22 & $90-105$ \\
Frosttage & 81 & $30-35$ \\
\hline Sommertage & 22 & 5.5. \\
\hline Mittlerer Beginn der Apfelblüte/Frühlingsbeginn & 25.4. & 40 \\
\hline Nebeltage & $50-70$ & $520-600 \mathrm{~mm}$ \\
\hline Niederschlag & $<800 \mathrm{~mm}$ & \\
\hline
\end{tabular}

Die Uckermark ist eine Region im Nordosten Brandenburgs (über $600 \mathrm{~km}$ Entfernung vom Saarland) zwischen Havel, Eberswalder Urstromtal und Oder an der Grenze zu Polen. Das betrachtete Gebiet in der Uckermark deckt sich mit den Außengrenzen des Biosphärenreservates "Schorfheide-Chorin", das verwaltungstechnisch den Kreisen Uckermark (im Norden) und Barnim (im Süden) zugeordnet ist. Die landwirtschaftlich und forstwirtschaftlich genutz- 
ten Flächen halten sich im $1290 \mathrm{~km}^{2}$ großen Untersuchungsgebiet von ihrer Ausdehnung her etwa die Waage; die Besiedlungsdichte mit weniger als 30 Einwohnern pro $\mathrm{km}^{2}$ ist für deutsche Verhältnisse außerordentlich gering.

Die untersuchte Landschaft wurde im Ergebnis der letzten Eiszeit (Pommersches Stadium der Weichselvereisung) geformt und weist über 200 größere natürliche Standgewässer auf (MAUERSBERGER \& MAUERSBERGER, 1996). Die Höhenlage der in dieser Arbeit genannten Seen liegt zwischen $25 \mathrm{~m}$ ü. NN (Gr. Aalgastsee) und $106 \mathrm{~m}$ ü. NN (Schwarzer See am Blocksberg).

\section{Methodik}

\subsection{Vorbemerkungen}

Der Schwerpunkt der Untersuchungen, die immerhin über die Hälfte aller rezent bekannten Fundgewässer der Art in Deutschland einbeziehen, lief zeitgleich in den Jahren 1992-1996 mit abgestimmter, mehrfach überarbeiteter bzw. geänderter Methodik in den beiden Untersuchungsregionen ab. Wo Unterschiede in Bezug auf Methodik oder Ergebnisse erwähnt werden müssen, wurden kennzeichnende Kürzel eingefügt: $(S)=$ Saarland; $(U)=$ Uckermark.

In den nachfolgenden ökologischen Betrachtungen wurden die ab 1996 entdeckten Fundorte im Saarland und im luxemburgischen Moseltal sowie die Veränderungen nach dem kalten Winter 1995/1996 in der Uckermark nicht berücksichtigt.

Einige Gewässer in beiden Gebieten, an denen trotz mehrjähriger Untersuchung Epitheca nicht nachgewiesen werden konnte, wurden vergleichend in die Erörterungen einbezogen, um Ausschlußkriterien für die Besiedlung herauszuarbeiten.

\subsection{Faunistische Untersuchungen: Libellen und Fische}

Die faunistische Erfassung des Vorkommens von Epitheca bimaculata begann bereits 1987 bzw. $1988 \mathrm{im}$ Vorfeld der hier dargestellten ökologischen Untersuchung, wurde aber bis 1996 fortgeführt. Hierzu wurden Exuvien teils stichprobenartig, teils systematisch aufgesammelt und Imagines beobachtet, wobei ein Fernglas wegen des Aktivitätsbereiches des Zweiflecks über dem offenen Wasser unabdingbar war. Vom Boot aus suchten wir nach Eigelegen (gallertige, an untergetauchten Pflanzenteilen hängende Stränge mit mehreren Hundert oder Tausend Eiern). Die faunistischen Ergebnisse müssen an anderer Stelle dargelegt werden (s. TROCKUR \& MAUERSBERGER in prep.).

Parallel dazu konnte das Vorkommen anderer Libellenarten als potentielle Konkurrenten und Indikatoren für den Zustand des Ökosystems mit erfaßt werden, wobei zur Ermittlung des Arteninventars auch Exkursionen - außerhalb der Epitheca-Flugzeit - von Juli bis September durchgeführt wurden. Die Angaben zur Libellenfauna basieren auf mindestens 3, meist jedoch wesentlich mehr Untersuchungstagen. Bei den meisten Gewässern liegen Informationen aus mehreren Untersuchungsjahren vor, so daß von einer recht guten Datenbasis ausgegangen werden kann.

Untersuchungen zur Besiedlung durch Fische mußten herangezogen werden, weil Fische einerseits für die Prädation von Larven von Bedeutung sein könnten, andererseits alle Stoffumsätze im Gewässer über die Fraßnahrungskette steuern und damit das Ökosystem gestalten. Die Fischfauna der saarländischen Untersuchungsgewässer ist nur lückenhaft bekannt. Bei fast allen Gewässern beruhen die vorliegenden Daten auf Angaben der örtlichen Angler bzw. meist zufälligen eigenen Beobachtungen. Die Güte der Daten vonseiten der Hobby-Angler ist erfahrungsgemäß unbefriedigend (mange Inde Kenntnis, teils auch beabsichtigt falsche/unvoll- 
ständige Angaben). Allerdings wird angenommen, daß der Kenntnisstand zu den in diese Überlegungen einbezogenen Arten als repräsentativ angesehen werden kann. Für einige der saarländischen Fundorte konnte auf durch Elektroabfischen gewonnene Ergebnisse im Rahmen von Pflege- und Entwicklungsplänen zurückgegriffen werden (ARGE IFÖNA ÖKOTOP, 1990), GfUG, 1995).

Die Angaben zum Fischbestand der uckermärkischen Gewässer stammen zumeist aus der Fischartenkartierung im Biosphärenreservat ,Schorfheide-Chorin“ (s. a. BURCKHARDT, 1993, KÄHLER mündl. 1995), die auf Zusammenarbeit mit den gewerblichen Fischereibetrieben und eigenen Untersuchungen beruht.

\subsection{Limnologische Untersuchungen: Morphometrie, Schichtung, Wasserbe- schaffenheit}

Für jedes der Gewässer haben wir (mit einem größeren Gegenstand, der wenig in weiche Sedimente einsinkt) die maximale Tiefe ausgelotet; außerdem wurden für einige Seen Querschnitte der Gewässerbodenlinie angefertigt. Tiefenprofile von Sauerstoffgehalt und Wassertemperatur an der tiefsten Stelle der Gewässer wurden in Schönwetterphasen im Sommer vom Boot aus aufgenommen (WTW Oxi-Meter 196 (U), bzw. 96 (S)).

Da sich im Laufe der Untersuchungen zeigte, daß die üblicherweise erhobenen wasserchemischen Parameter für Epitheca bimaculata wohl wenig relevant sind, wurde auf ein breites Erhebungsspektrum verzichtet. Lediglich die Karbonathärte als Maß für den Bicarbonatgehalt - ermittelt mit Testreagenzien der Firma Merck - soll hier stellvertretend als Parameter der Ionenversorgung des Wassers und als pH-Wert-Puffer aufgeführt werden.

Zur groben trophischen Kennzeichnung wurden möglichst oft Sichttiefenmessungen an den Gewässern durchgeführt. Für die uckermärkischen Seen konnte in den meisten Fällen bereits auf vorhandene Daten zurückgegriffen werden (MAUERSBERGER \& MAUERSBERGER 1996).

\subsection{Erfassung von Habitatstrukturen: Vegetation, Sedimente, Umfeld}

Da erwartet wurde, daß die Eignung als Bruthabitat für Epitheca maßgeblich von der Strukturierung der Wasserkörper abhängt, wurde am Gewässer das Vorkommen von Pflanzenbeständen notiert und ihre Ausdehnung abgeschätzt.

- Wasserröhrichte: im Wasser stehende Bestände von Schilf, Rohrkolben und Teichsimse (Phragmites australis, Typha spp., Schoenoplectus lacustris);

- Wasserrieder: im Wasser stehende Seggen, Binsen und andere niedrigere Strukturen (Carex spp., Juncus spp., Cladium mariscus, Eleocharis palustris u. a.);

- Schwingkantenrieder (nur U): Riedvegetation der wasserseitigen Kanten schwimmender Torfkörper (bestehend vor allem aus Carex spp., Thelypteris palustris, Cladium);

- Schwimmblattrasen: im Sediment wurzelnde, auf der Wasseroberfläche assimilierende Pflanzen (Nuphar lutea, Nymphaea alba, Potamogeton natans, Polygonum amphibium);

- oberflächennahe Submerse (Tauchfluren und Schwebematten bestehend aus Myriophyllum spp., Ceratophyllum spp., Potamogeton spp., Utricularia spp.);

- Submersvegetation tieferer Schichten (Grundrasen und Grundmatten bestehend aus Characeen, Ceratophyllum demersum, Utricularia vulgaris);

- Totholz: im Wasser liegende oder stehende Stämme, Äste und Zweige;

- Ufergehölze: im Wasser stehende (Alnus, Salix) oder oberhalb der Wasserlinie wurzelnde und das Gewässer z. T. beschattende Büsche und Bäume.

Bei der Suche nach Submersmakrophyten kam ein „Krautanker“ zum Einsatz. 
Da durch Aquarium- und Kescherversuche die Sedimente als Larvenhabitat angesprochen werden konnten, wurden diese in die Untersuchungen einbezogen. Die Kenntnisse über die Art der ufernahen Sedimente (Unterwasserböden) ergaben sich einesteils aus Larvenfangversuchen mit Hilfe eines Sedimentkeschers oder eines Küchensiebes, andernteils aus der visuellen Prüfung und der Begehung der Flachwasserbereiche oder aus Sedimentbohrungen (U) an den von der Art besiedelten Gewässerabschnitten.

Zur groben Charakterisierung des Umfeldes wurde aus Luftbildern und der eigenen Anschauung vor Ort die Wald-Offenland-Verteilung ermittelt. Auch das Ausmaß der Bestockung mit Ufergehölzen wurde u. a. im Hinblick auf den Einfluß auf die Sedimentbildung bzw. auf die mikroklimatischen Bedingungen derart erfaßt.

\subsection{Klassifikation des Status der Gewässer für Epitheca bimaculata}

In Anlehnung an STERNBERG (1990) wurde eine Statuseinteilung der Fundorte in 3 Gruppen (Tab. 2) vorgenommen, wobei die Bedeutung des Begriffes Latenzhabitat abgewandelt, die Bezeichnung jedoch übernommen wurde. Diese Dreiteilung wird für parameterbezogene Auswertungen in den folgenden Kapiteln benutzt, um eine Wichtung der Ergebnisse zu ermöglichen: in Stammhabitaten liegt eine Merkmalskombination vor, die offenbar die Ansprüche der Art optimal repräsentiert.

Tab. 2: Definition des Status der Epitheca-Fundorte.

\begin{tabular}{|l|l|l|}
\hline Bezeichnung & Bedeutung & Abgrenzung \\
\hline Stammhabitat & $\begin{array}{l}\text { Gewässer mit hoher Exuvienabundanz } \\
\text { und Populationsdruck }\end{array}$ & $\begin{array}{l}\text { mindestens 10 Exuvien in einem Jahr } \\
\text { gefunden }\end{array}$ \\
\hline Nebenhabitat & $\begin{array}{l}\text { suboptimales Fortpflanzungsgewässer } \\
\text { mit nur geringer Exuvienabundanz }\end{array}$ & $\begin{array}{l}\text { stets weniger als 10 Exuvien in einem } \\
\text { Jahr }\end{array}$ \\
\hline Latenzhabitat & $\begin{array}{l}\text { Gewässer, das von Imagines aufgesucht } \\
\text { wird, ein Reproduktionsnachweis fehlt } \\
\text { aber bisher }\end{array}$ & nur Imagines beobachtet \\
\hline
\end{tabular}

In beiden Regionen ist jeweils ein Viertel der gut untersuchten Fundorte als Stamm- bzw. Latenzhabitat einzuordnen, etwa die Hälfte sind Nebenhabitate (s. Tab. 9).

Die beiden Fundorte mit den höchsten Exuvienabundanzen (Dreiecksee, Oberer Altarm Beckingen) gelten zusammen mit dem individuenstärksten Fundort in den französischen Ardennen (COPPA, 1991) als die "besten" Epitheca-Gewässer in Mitteleuropa. Darüberhinaus sind bisher nur wenige abundanzstarke Epitheca-Vorkommen bekannt (VINCENT u. a., 1987: Lothringen, KOTARAC, in litt.: Slowenien).

\section{Ergebnisse}

\subsection{Gewässertyp, Hydrologie und Genese}

In Tabelle 3 werden die Gewässertypen der Epitheca-Fundorte in beiden Regionen verglichen. Hydrologisch gesehen sind die Unterschiede zwischen den Epitheca-Gewässern in Saarland und Uckermark recht groß, noch mehr weichen sie allerdings in ihrer Genese voneinander ab. Die Altwässer, Fisch- und Kiesteiche im Saarland werden vom Grundwasser der Auen gespeist bzw. besitzen mehr oder weniger starken Fließgewässereinfluß. 
Tab. 3: Übersicht über die Typen der von Epitheca besiedelten Gewässer.

\begin{tabular}{|l|c|c|}
\hline Gewässertyp & Uckermark & $\begin{array}{c}\text { Saarland und angrenzendes } \\
\text { Luxemburg }\end{array}$ \\
\hline Natürliche Seen & 48 & 26 \\
\hline Abgrabungsgewässer (Kiesgruben) & & 6 \\
\hline Fischteiche & 1 & 7 \\
\hline Altarme/Altwässer & & \\
\hline
\end{tabular}

Unter den uckermärkischen Fundorten befindet sich lediglich ein Teich und kein einziges Auengewässer; vielmehr handelt es sich überwiegend um Seen, von denen über die Hälfte oberirdisch unverbunden ist und auch nur selten intensiven Grundwasserkontakt besitzt. Von der Genese her stehen künstlich geschaffene Flußaltarme (besser: Altwässer) und Abgrabungen des Menschen mit z. T. sehr geringem Alter im Saarland den eiszeitlich geformten, zum Teil etwa 10000 Jahre alten Seen der Uckermark (vor allem ehemalige Toteislöcher) gegenüber.

\subsection{Gewässerphysiognomie/Morphometrie}

\subsubsection{Ausdehnung der Wasserfläche}

Die kleinsten Gewässer, die von Epitheca angeflogen werden, besitzen sowohl im Saarland (Unterer Altarm Beckingen 2, Altarm Rehlingen 3) wie auch in der Uckermark (Barschpfuhl, Kleiner Kagelpfuhl) Größen von ca. 0,5 Hektar (50 x $100 \mathrm{~m})$; auch erfolgreiche Reproduktion konnte hier nachgewiesen werden.

Die größten Epitheca-Gewässer sind der Dillinger Saar-See (20 ha), der Jakobsdorfer See (23,5 ha) und der Redernswalder See (48 ha). In diesen größeren Gewässern gehören aber jeweils nur einzelne Uferstrecken oder Buchten, die die notwendigen Strukturen bieten, zum Epitheca-Habitat.

Aus Figur 2 geht hervor, daß die Wasserfläche bei über der Hälfte (68\%) der Epitheca-Habitate kleiner als 5 ha ist; Gewässer größer als 15 ha werden nur ausnahmsweise besiedelt.

Für die Graphik in Figur 3 wurden nur die Fundgewässer mit einer Fläche unter 10 ha herangezogen, um eine genauere Differenzierung innerhalb des präferierten Bereiches erkennen zu können. Es zeigt sich, daß wiederum die Gruppe der kleinsten Gewässer ( 0 - 3 ha) von vorrangiger Bedeutung ist.

Noch deutlicher wird die Aussage, wenn man nur die Optimalgewässer herausgreift: mit einer Ausnahme bewegt sich ihre Oberfläche zwischen 1,2 -7,7 ha. Der kürzeste Durchmesser dieser Gewässer beträgt nur 50 bis $200 \mathrm{~m}$. Aus Figur 4 wird deutlich, daß bei den Neben- und v. a. bei den Latenzhabitaten die Streuung weitaus größer ist als bei den Stammhabitaten. Der Mittelwert bei Stamm- und Nebenhabitaten liegt jeweils bei etwa 5 ha.

\subsubsection{Tiefe der Wasserkörper}

Die Präferenz von Epitheca bimaculata für Seen bestimmter Tiefe wird vor allem dann deutlich, wenn man die Tiefe aller vorhandenen Seen des Gebietes dazu in Beziehung setzt. Für das uckermärkische Untersuchungsgebiet wurde hierfür die Datenbasis aus MAUERSBERGER \& MAUERsBerger (1996) herangezogen. Da die Tiefe des Gewässers für die Imago nicht wahrnehmbar ist, wurde für die nachfolgende Darstellung (Fig. 5) auf die Gewässer, an denen keine Exuvien- oder Larvenfunde vorliegen, verzichtet. 

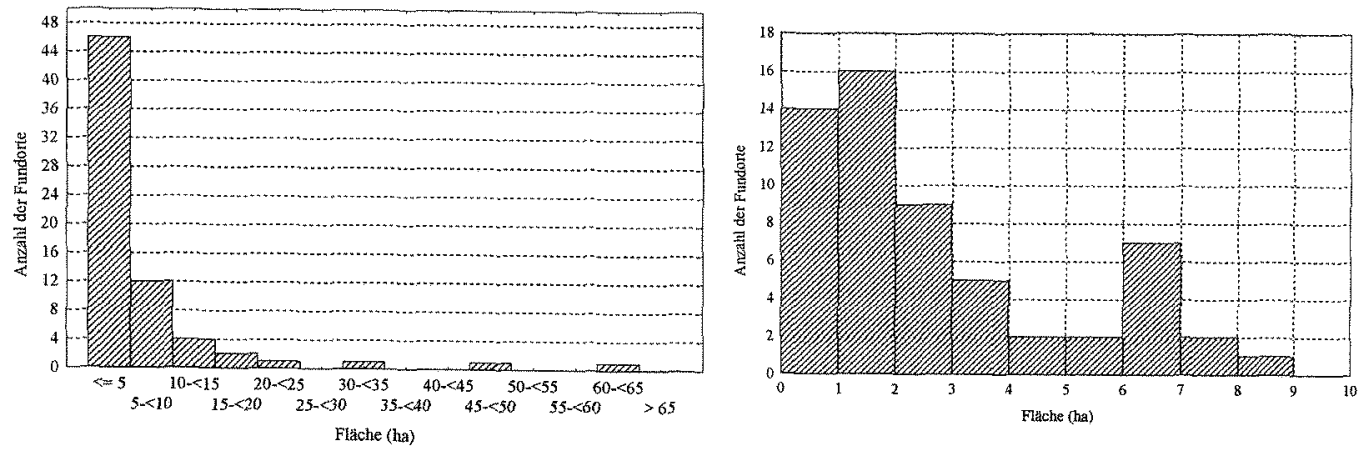

Fig. 2: Größe der Wasserfläche der Epitheca-Gewässer in Saarland und Uckermark. - Fïg. 3: Größenverteilung der kleineren Epitheca-Gewässer (unter 10 ha, $\mathrm{n}=57$ ).
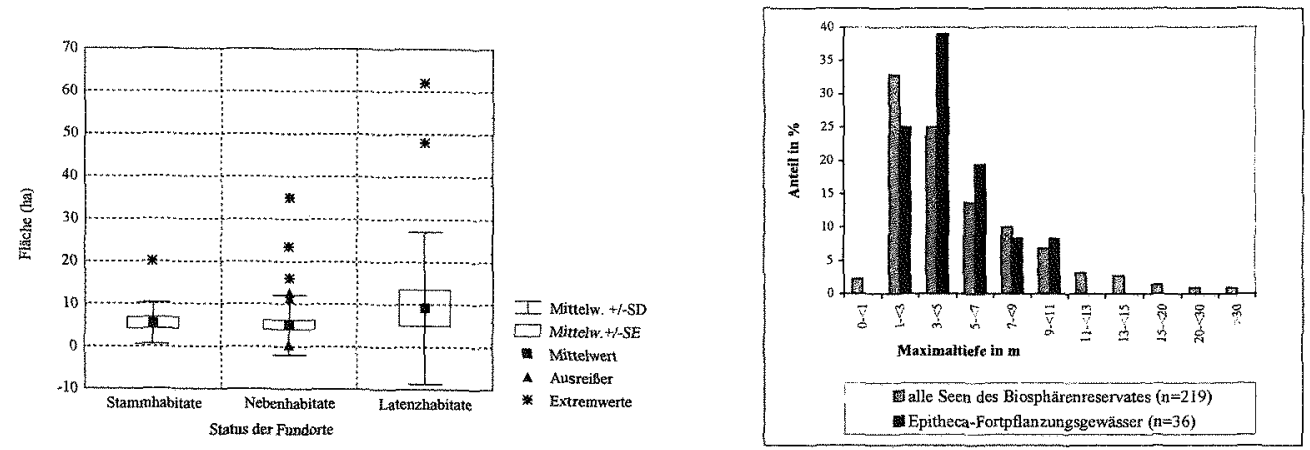

Fig. 4: Größenvergleich der Gewässer nach ihrem Status. - Fig. 5: Tiefenstufen der Epitheca-Seen in der Uckermark im Vergleich zur naturräumlichen Ausstattung.

Fig. 6: Vergleich der Maximaltiefe nach dem Fundortstatus (beide Gebiete).

Figur 5 läßt erkennen, daß sowohl die ausgesprochenen Seichtseen mit Maximaltiefen unter $1 \mathrm{~m}$ sowie die Seen tiefer als $11 \mathrm{~m}$ generell nicht von Epitheca besiedelt werden. Seen mit einer maximalen Tiefe zwischen 3 und $7 \mathrm{~m}$ werden hingegen deutlich

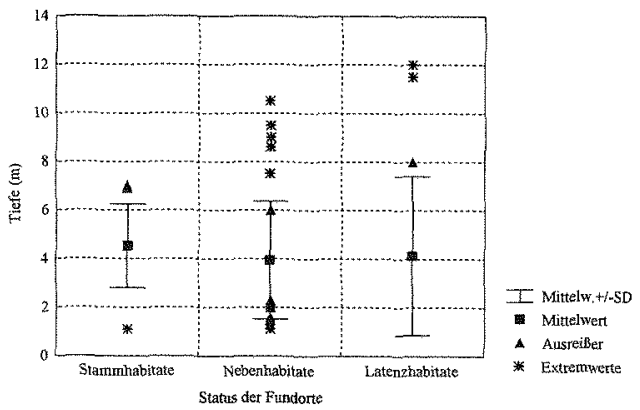
häufiger als Brutgewässer angenommen, als es bei einer Gleichverteilung auf alle Tiefenstufen wahrscheinlich wäre. In diesen Gewässern sind gute Bedingungen für eine erfolgreiche Fortpflanzung der Art gegeben.

Daß tatsächlich eine Präferenz für bestimmte Tiefenstufen besteht, zeigt Figur 6: die Stammhabitate sind deutlich stärker eingeengt mit einem Maximum zwischen 3 und $6 \mathrm{~m}$ Tiefe. Die Gegenüberstellung der saarländischen und uckermärkischen Fundgewässer zeigt eine deutlich höhere Streuung und einen Mittelwert bei 4,5 $\mathrm{m}$ in der Uckermark. Im Saarland weisen Epitheca-Gewässer im Mittel eine Tiefe von nur $3 \mathrm{~m}$ auf. Dieser Unterschied spiegelt allerdings die unterschiedliche naturräumliche Ausstattung der Gebiete wider. 
Bei separater Betrachtung nur der Optimalgewässer wird erkennbar, daß die Stammgewässer des Saarlandes deutlich tiefer als die anderen und damit denen der Uckermark ähnlicher sind (s. Tab. 4).

Tab. 4: Maximaltiefe von Epitheca-Gewässern (Mittelwerte).

\begin{tabular}{|l|c|c|}
\hline & Saarland & Uckermark \\
\hline Stammhabitate & $3,9 \mathrm{~m}$ & $4,8 \mathrm{~m}$ \\
\hline Neben- und Latenzhabitate & $2,7 \mathrm{~m}$ & $4,5 \mathrm{~m}$ \\
\hline
\end{tabular}

An zahlreichen Zweifleck-Stammhabitaten in beiden Regionen wurden Unterwasserbodenprofile zur Ermittlung der Neigung des Gewässerbodens angefertigt. Mehrfach wurde ufernah ein relativ steiler Abfall auf Tiefen von 2 bis fast $4 \mathrm{~m}$ festgestellt.

Daß dieser Parameter für Epitheca keine Bedeutung besitzt, wird klar, wenn man Gewässer wie den Liptowsee mit einer Maximaltiefe von nur 1,1 m betrachtet. Aus diesem Grunde haben wir auf eine vollständige Darstellung und Auswertung unserer diesbezüglichen Meßergebnisse verzichtet.

\subsection{Temperatur- und Sauerstoffschichtung des Wasserkörpers}

Die Variabilität in der Temperatur- und Sauerstoffschichtung zwischen den einzelnen Fortpflanzungsgewässern ist beträchtlich. Da Epitheca keine Präferenz zeigte, wurde auf eine Darstellung und Auswertung der Tiefenprofile verzichtet.

Vollständig geschichtete Seen mit einer sigmoiden Temperaturkurve im Sommer wurden unter den Epitheca-Gewässern nur in der Uckermark und auch dort nur in geringer Zahl gefunden. Die meisten Wasserkörper besitzen lediglich in Grundnähe eine deutlich geringere Temperatur als an der Oberfläche, wobei der Temperaturabfall in die Tiefe steil klinal (vielfach im Saarland) oder sprunghaft (vor allem in der Uckermark) verläuft. Auffälligerweise besitzen auch die besonders seichten Gewässer (z. B. Liptowsee, Krummer See/Liepe, Moossee) im Sommer wegen der Trübung oder durch Huminstoffe verursachten Färbung eine deutlich niedrigere Temperatur am Grunde als an der Wasseroberfläche.

Tiefenprofilmessungen des Sauerstoffgehaltes während der Sommerstagnation haben ergeben, daß die Seen mit nachweislich bodenständigen Epitheca-Vorkommen bei spätestens $5,9 \mathrm{~m}$ Tiefe sauerstofffreies Wasser führen, bei einigen treten anaerobe Verhältnisse bereits unterhalb von 2,7 m (U) bzw. 1,5 m (S) auf. Im Brackensee (U) lag der Sauerstoffgehalt schon bei 1,7 m Tiefe deutlich unter dem Wert an der Oberfläche. An einigen saarländischen Gewässern ist ein starker Abfall der $\mathrm{O}_{2}$-Konzentration schon bei weniger als einem Meter zu verzeichnen.

\subsection{Wasserbeschaffenheit}

\subsubsection{Säure-Basen-Stufe und Wasserhärte}

Das Gros der Epitheca-Gewässer ist alkalisch mit pH-Werten zwischen 7 und 9; die beiden einzigen Ausnahmen befinden sich in der Uckermark (Hechtdiebel und Liptowsee). Hier sinken die $\mathrm{pH}$-Werte vor allem im Winter mitunter bis auf 5,5 ab.

Da der $\mathrm{pH}-$ Wert besonders bei belasteten Seen bereits im Tagesgang enormen Schwankungen unterworfen sein kann, haben wir auf eine Darstellung in Tabelle 11 verzichtet. Statt dessen beziehen wir uns auf den Kalkgehalt, der sich recht gut indirekt über die Karbonathärte wiedergeben läßt. 
Die Spanne der Werte von $0,2^{\circ}$ bis $16^{\circ} \mathrm{dH}$ (ähnlich bei der Leitfähigkeit: 0,04 bis 0,75 $\mathrm{mS} / \mathrm{cm}^{2}$ ) ist enorm; lediglich extrem weiche und damit saure Gewässer werden nicht besiedelt (s. Kap. 5.7.1).

\subsubsection{Wassertransparenz und Trophie}

Direkte Produktions- oder Nährstoffparameter wurden nicht gemessen, nur für einige der Gewässer sind uns Laboranalysen bekannt geworden, die aber in aller Regel unsere Einschätzungen, die auf Messungen der Sichttiefe und der Erfassung der submersen Vegetation beruhen, bestätigen.

Die Sichttiefe gibt eine zeitlich variable Trübung durch Phytoplankton und suspendierte Sedimentpartikel wieder. Das sommerliche Mittel der Sichttiefe von Epitheca-Gewässern nimmt Werte zwischen 0,5 m und 3,9 m an, Stammhabitate weisen Sichttiefen zwischen 0,5 und 2,4 m auf. Aus Tabelle 5 geht hervor, daß die saarländischen Epitheca-Gewässer deutlich trüber sind als die uckermärkischen bei großen Unterschieden zwischen den Latenzhabitaten, jedoch nur geringen Abweichungen bei den Stammhabitaten.

Tab. 5: Durchschnitt der Mittleren Sichttiefen der Epitheca-Gewässer.

\begin{tabular}{|l|c|c|c|c|c|c|c|c|c|}
\hline & \multicolumn{4}{|c|}{ Saarland } & \multicolumn{3}{c|}{ Uckermark } & \multicolumn{3}{c|}{$\begin{array}{c}\text { beide } \\
\text { Regionen }\end{array}$} \\
\hline Status von Epitheca & $\mathrm{n}$ & $\begin{array}{c}\text { mittl. } \\
\text { Sichttiefe } \\
(\mathrm{m})\end{array}$ & $\mathrm{s}$ & $\mathrm{n}$ & $\begin{array}{c}\text { mittl. } \\
\text { Sichttiefe } \\
(\mathrm{m})\end{array}$ & $\mathrm{s}$ & $\mathrm{n}$ & $\begin{array}{c}\text { mittl. } \\
\text { Sicht- } \\
\text { tiefe (m) }\end{array}$ & $\mathrm{s}$ \\
\hline Stammhabitate & 4 & 1,4 & 0,45 & 9 & 1,7 & 0,60 & 13 & 1,6 & 0,58 \\
\hline Nebenhabitate & 10 & 1,1 & 0,43 & 27 & 1,7 & 0,68 & 37 & 1,5 & 0,68 \\
\hline Latenzhabitate & 5 & 0,9 & 0,47 & 12 & 1,9 & 0,72 & 18 & 1,6 & 0,9 \\
\hline alle Habitate & 19 & 1,1 & 0,48 & 49 & 1,7 & 0,71 & 68 & 1,6 & 0,72 \\
\hline
\end{tabular}

Im Durchschnitt der sommerlichen Sichttiefen weist Tabelle 5 1,6 Meter als Durchschnitt bei allen Fundorten wie auch bei Fundorten mit höheren Reproduktionszahlen bei Epitheca aus; die anderen Gewässer (von Epitheca dünn oder nicht besiedelt) sind im Saarland trüber und in der Uckermark etwas klarer.

Die mittlere Sichttiefe an jedem Stammhabitat bleibt an Saar und Ucker unter 2,5 m, obwohl im uckermärkischen Untersuchungsgebiet 35 Seen mit Sichttiefenmitteln zwischen 2,5 und 6,7 $\mathrm{m}$ existieren (mesotrophe Klarwasserseen, s. Kap. 5.7: Negativgewässer).

Die trophische Situation von Epitheca-Gewässern bewegt sich zumeist im eutrophen bis polytrophen Niveau (Klassifikation in Anlehnung an TGL, 1982 bzw. LAWA, 1999); der einzige mesotrophe See darunter (K1. Vätersee, Uckermark) ist nur Latenzgewässer.

\subsection{Habitatstrukturen}

\subsubsection{Vegetationsstrukturen am Wasser}

Sowohl als Teil des Imaginallebensraumes als auch als Einflußgröße des Larvenhabitates sind Ufergehölze und Wälder in der Nähe des Gewässers von Interesse.

Auf den ersten Blick scheinen sich die Bedingungen an den Epitheca-Gewässern von Saarland und Uckermark in Bezug auf diese beiden Parameter deutlich zu unterscheiden. Aus Tabelle 6 geht hervor, daß in der Uckermark nur jeder zweiter See über ausgeprägte Ufergehölze an 
der Wasserlinie verfügt, während im Saarland fast jedes Gewässer Gehölze am Ufer besitzt. Die uckermärkischen Epitheca-Seen liegen dafür fast durchweg im Wald, was im Saarland eher die Ausnahme darstellt. Werden jedoch beide Merkmale zusammengefaßt, so ergibt sich, $\mathrm{da} ß$ in den untersuchten Regionen nahezu alle Gewässer von Gehölzbeständen umgeben sind.

Tab. 6: Ausstattungsgrad von Epitheca-Gewässern mit Gehölzstrukturen.

\begin{tabular}{|l|c|c|c|}
\hline & Saarland & Uckermark & gesamt \\
\hline $\begin{array}{l}\text { Ufergehölze } \\
\text { (mit 2 und 3 klassifizierte Vorkommen aus Tab. 11 } \\
\text { berücksichtigt) }\end{array}$ & $84 \%$ & $43 \%$ & $55 \%$ \\
\hline $\begin{array}{l}\text { gewässernaher Wald } \\
\text { (mit 3 und 4 klassifizierte Bestände aus Tab. 11 be- } \\
\text { rücksichtigt) }\end{array}$ & $\mathbf{1 6 \%}$ & $96 \%$ & $74 \%$ \\
\hline Ufergehölze oder Wald vorhanden & $\mathbf{8 4 \%}$ & $\mathbf{1 0 0 \%}$ & $\mathbf{9 6 \%}$ \\
\hline
\end{tabular}

Weiterhin ist hervorzuheben, daß es sich generell überwiegend um Laubgehölze handelt, die an den Gewässern vorgefunden wurden (s. Fig. 7). Die Ausnahmen betreffen Fundorte, an denen die Art nicht oder nicht regelmäßig bodenständig ist.

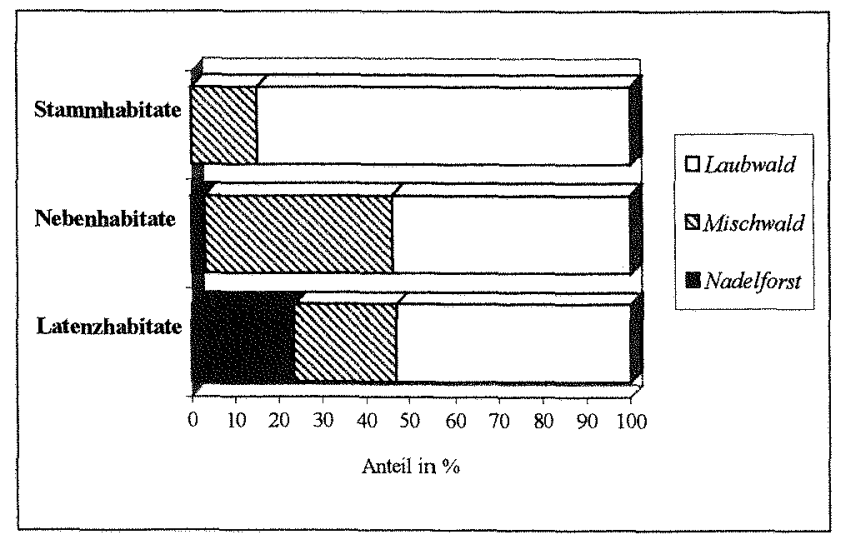

Fig. 7: Art der Bestockung in Waldflächen an Epitheca-Gewässern (Uckermark + Saarland).

\subsubsection{Vegetationsstrukturen im Wasser}

Die Vegetationsstrukturen im Wasserkörper wurden nach Lebensformtypen differenziert (Definitionen in Kap. 3.4) erfaßt.

Typisch für Epitheca-Gewässer sind Pflanzenstrukturen an der Wasseroberfläche, die in der Uckermark zumeist von Schwimmblattrasen, im Saarland häufiger von oberflächennahen Unterwasserpflanzen gebildet werden (s. Tab. 8).

Weiterhin haben die Gewässer in beiden Regionen gemeinsam, daß Riede deutlich häufiger anzutreffen sind als Großröhrichte. Keines der Stammhabitate besitzt beispielsweise einen bedeutenden Röhrichtgürtel von etwa 10 oder $20 \mathrm{~m}$ Breite.

Schwingkantenstrukturen wurden nur in der Uckermark angetroffen; an den jungen Gewässern im Saarland fehlen derartige Bildungen. Auch die Vorkommen von Totholzstrukturen sind in der Uckermark stärker, wo abflußlose Seen existieren, die eine starke langfristige Pegeldyna- 


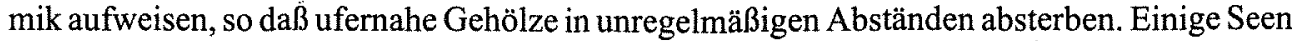
wie z. B. der Rohrhahngrund werden von toten Baumstämmen, Stubben, Ästen und Zweigen in der Uferzone geprägt.

Die geringere Pegeldynamik sorgt reziprok im Saarland für die häufige Ausbildung von Ufergehölzstreifen (s. 4.5.1.).

Tab. 7: Vegetationsstrukturen an den untersuchten Gewässern (Datenbasis s. Tab. 11 in Kap. 4.7.; als vorhanden gewertet wurden für diese Auswertung die Häufigkeitsklassen 2 - 4).

\begin{tabular}{|l|c|}
\hline Strukturtyp & Präsenz \\
\hline Schwimmblattrasen & $77 \%$ \\
\hline oberflächennahe Submersvegetation & $49 \%$ \\
\hline Gesamt: Schwimmblatt oder oberflächennahe Submerse & $\mathbf{9 0 \%}$ \\
\hline Submersvegetation in größerer Tiefe & $57 \%$ \\
\hline Wassergroßröhrichte & $47 \%$ \\
\hline Wasserriede & $90 \%$ \\
\hline
\end{tabular}

Tab. 8: Dominante Vegetationsstrukturen im Wasser an den Epitheca-Stammhabitaten (Bezeichnung der Gewässer s. Tab. 9 und 11).

Bedeutung der Signaturen:

flächig und aspektbildend ausgebildet
kleinflächig oder lokal
nur in Einzelexemplaren vorhanden
fehlend

\begin{tabular}{|c|c|c|c|c|c|c|c|c|c|c|c|c|c|}
\hline Stammhabitate: & \multicolumn{4}{|c|}{ Saarland } & \multicolumn{9}{|c|}{ Uckermark } \\
\hline Vegetationsstruktur: & A & B & $\mathbf{E}$ & $G^{*}$ & 5 & 37 & 12 & 15 & 16 & 26 & $10 *$ & 36 & 47 \\
\hline \multirow{2}{*}{\multicolumn{14}{|c|}{$\begin{array}{l}\text { Nuphar lutea } \\
\text { Potamogeton natans }\end{array}$}} \\
\hline & & & & & & & & & & & & & \\
\hline \multicolumn{14}{|l|}{ Nymphaea alba } \\
\hline \multicolumn{14}{|l|}{ Myriophyllum spp. } \\
\hline \multicolumn{14}{|l|}{ Ceratophyllum demersum } \\
\hline \multicolumn{14}{|l|}{ Elodea canadensis/nutallii } \\
\hline \multicolumn{14}{|l|}{ andere Submerse } \\
\hline \multicolumn{14}{|l|}{ Phragmites australis } \\
\hline \multicolumn{14}{|l|}{ Typha spp. } \\
\hline \multicolumn{14}{|l|}{ Schwingkantenstrukturen } \\
\hline Totholz & & & & & & & & & & & & & \\
\hline
\end{tabular}

*: Situation bis 1993 (Saarland G), bis 1996 (Uckermark 10) 
In Tabelle 8 werden anhand der Stammgewässer die vorgefundenen Kombinationen von Vegetationstrukturen, die für die Eiablage und das Larvenhabitat relevant sind, dokumentiert. Besonders häufig sind Nuphar und Myriophyllum an der Bildung der entscheidenden Strukturen beteiligt. Die Muster der realisierten Habitatansprüche sind insgesamt recht unterschiedlich.

\subsubsection{Sedimente (Seeboden)}

Aufgrund von Aquariumbeobachtungen und Kescherversuchen läßt sich bezüglich der Sedimente zumindest für die älteren Larvalstadien eine Bedeutung als Lebensraum annehmen.

Besonders im Saarland steht als Ausdruck des geringen Alters der Gewässer vielfach noch der kahle mineralische Untergrund (Lehm, Kies) an. Höhere Exuvienabundanzen der Art wurden jedoch nur an Ufern nachgewiesen, wo lokal eine dünne organische Auflage (z. B. Blätter, Röhrichtteile, Holzstückchen, feines Astwerk vermengt mit deren Zersetzungsprodukten verschiedener Stadien, sonstiges Detritusmaterial) vorhanden ist.

Als Untergrund der meisten Seen (U) stehen im Ergebnis jahrtausendelanger Sedimentationsprozesse Mudden (,Schlamm“ oder Gyttja, Zersetzungsprodukt vor allem limnischer Organismen) in einer Mächtigkeit von mehreren Metern an. Vornehmlich werden die tieferen, zentralen Gewässerbereiche von Mudden ausgekleidet. Seen, in denen die weichen Sedimente auch im Uferbereich anzutreffen sind, bringen jedoch nur geringe Epitheca-Abundanzen hervor (Nebenhabitate).

Einen besonderen Fall repräsentiert der Liptowsee. An seinem Ostufer stehen mineralische Sedimente mit dünner Auflage an, den Rest des Seebodens bilden Mudden. Jedoch wurden am Nordufer mit zahlreichen Exuvienfunden bis 20 Meter see-einwärts in der oberen Muddeschicht noch ein festes Geflecht abgestorbener Rhizomteile (,Unterwassertorf“, subaquatischer Torf in sensu SuCCOW, 1988) von Equisetum und Phragmites gefunden.

\subsection{Begleitfauna}

\subsubsection{Fischfauna}

In allen Epitheca-Habitaten kommen Fische vor: zwischen 1 und 16 Fischarten wurden während der letzten Jahre nachgewiesen. Bei der schlechten Datenlage zur Fischfauna im Saarland beziehen sich die nachfolgenden Angaben in der Hauptsache auf die uckermärkischen Seen. Zum typischen Arteninventar der Gewässer gehören in erster Linie Plötzen (Rutilus rutilus), Rotfedern (Scardinius erythrophthalmus), Barsche (Perca fluviatilis), Hechte (Esox lucius), Schleie (Tinca tinca), Karauschen (Carassius carassius) und Bleie (Abramis brama) (S + U); ferner gehören in der Uckermark auch Ukelei (Alburnus alburnus), Güster (Blicca bjoerkna), Stichling (Gasterosteus aculeatus), Kaulbarsch (Gymnocephalus cernua) und Wels (Silurus glanis) zur autochthonen Fischfauna. Einige weitere Arten gelangten nur durch künstlichen Besatz in die Untersuchungsgewässer, wie z. B. Karpfen (Cyprinus carpio), Giebel (Carassius auratus f. gibelio), Kleine Maräne (Coregonus albula)(U), Zander (Stizostedion lucioperca) und Aal (Anguilla anguilla). Erst in den letzten 20-30 Jahren wurden auch pflanzenfressende Fischarten wie Silberkarpfen (Hypophthalmichthys molitrix), Weißer Amur (Ctenopharyngodon idella) und Marmorkarpfen (Aristichthys nobilis) aus Ostasien eingeführt.

Bei der Übersicht zur Fischfauna in Tabelle 11 wurde auf die Darstellung des Vorkommens der am Gewässergrunde nach Nahrung suchenden Arten sowie der Pflanzenfresser besonderer 
Wert gelegt; tatsächlich weist die Tabelle bei den am dichtesten mit Epitheca besiedelten Seen (U) das Fehlen (oder ein abundanzschwaches Vorkommen) des Karpfens und der Pflanzenfresser aus, auch die Bestände von Bleien, Karauschen und Güstern sind schwächer ausgeprägt als in anderen Seen. Die häufigsten Fischarten stellen Plötze, Rotfeder, Barsch, Schlei und Hecht dar.

Die meisten Gewässer werden durch Freizeitfischer genutzt. In der Uckermark werden 17 vor allem größere Epitheca-Seen von Fischereibetrieben im Haupterwerb bewirtschaftet. Mit der Ausweisung des Biosphärenreservates, ,Schorfheide-Chorin" erhielten einige Teilflächen einen Totalreservatsstatus, in denen auch unverhältnismäßig viele Epitheca-Gewässer liegen. So dürfen 11 Seen, darunter Kespersee, Rohrhahngrund, Brackensee, Wildbergsee und Moossee, die vor allem von Anglern bewirtschaftet wurden, seit 1990 nicht mehr genutzt werden.

\subsubsection{Libellen}

Würde man alle Libellen zusammenzählen, die an mindestens einem der untersuchten Epitheca-Gewässer von uns festgestellt wurden, so käme man auf 48 Arten. Diese Anzahl resultiert aus der Strukturvielfalt einiger Gewässer; eine bedeutende Artenfülle ist keinesfalls typisch für Epitheca-Habitate. Nur wenige Arten kommen an mehr als einem Viertel der Gewässer vor. Fast stetig sind vertreten: Ischnura elegans, Cordulia aenea und Orthetrum cancellatum. Orthetrum und Cordulia sind an allen Epitheca-Stammhabitaten bodenständig.

An uckermärkischen Seen annähernd durchweg vorhanden sind Erythromma najas, Aeshna grandis und Coenagrion pulchellum, die jedoch im Saarland seltener bzw. sehr selten sind. An saarländischen Epitheca-Gewässern wurden häufig Anax imperator und Gomphus pulchellus nachgewiesen, die in der Uckermark unstetig auftreten oder gänzlich fehlen. Erythromma viridulum wurde an vielen der Gewässer beobachtet; ob sich die Art darin auch reproduziert, ist jedoch zumeist unklar.

Unter den in Mitteleuropa weit verbreiteten und häufigen Arten fällt insbesondere die Seltenheit von Aeshna cyanea auf, von der nur an 3\% der untersuchten Gewässer ein Bodenständigkeitsnachweis erbracht werden konnte.

Obwohl nach JACOB (1969) Epitheca mit Anax parthenope angeblich vergesellschaftet sein soll, konnten wir letztere Art an Stamm- und Nebenhabitaten von Epitheca nicht nachweisen.

\subsection{Zusammenfassende Darstellung der Ergebnisse}

Aus der während der Untersuchungen mehrfach erweiterten Liste von Untersuchungsgewässern und von Parametern ergab sich eine schwer überschaubare Datenfülle. Da wir einen vergleichenden Überblick über diese Daten für unbedingt notwendig hielten, um die komplexe Merkmalskonstellation von Epitheca-Habitaten erkennbar werden zu lassen, haben wir uns entschlossen, sie in zwei großen Tabellen zusammenfassend darzustellen. Bei vielen Parametern mußten die Primärdaten in ein Klassifikationsschema gebracht werden, um eine Vergleichbarkeit zu ermöglichen. Nachfolgend werden die abgegrenzten Stufen innerhalb der einzelnen Merkmale definiert.

\subsubsection{Erklärungen für Tabelle 9}

Die Tabelle 9 enthält faunistische Daten sowie limnologische Angaben. 


\begin{tabular}{|c|c|c|c|c|c|c|c|c|c|c|c|c|c|c|c|c|}
\hline \multirow[t]{3}{*}{$\mathrm{Nr}$. } & \multirow[t]{3}{*}{ Fundort } & \multirow{2}{*}{\multicolumn{6}{|c|}{ Status v. E. bimaculata }} & \multirow{2}{*}{\multicolumn{8}{|c|}{ Limnologische Parameter }} & \multirow{3}{*}{$\mid$} \\
\hline & & & & & & & & & & & & & & & & \\
\hline & & 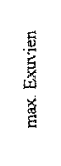 & 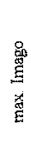 & 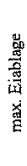 & 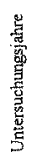 & 䔅 & 蛋 & 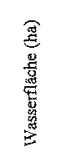 & 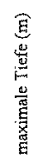 & 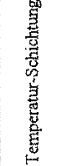 & $\begin{array}{l}\text { Sauerstoff: } \\
\text { Tiefe }(\mathrm{m}) \text { bei } \\
\text { Beginn der } \\
\text { Sprungschicht } \\
\text { und bei } 2 \mathrm{mg} /\end{array}$ & 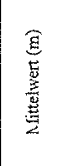 & 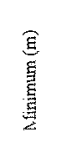 & 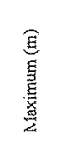 & 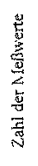 & \\
\hline 5 & Dreicoksee & 394 & 4 & 2 & 6 & 6 & II & 3,4 & 5 & $\mathrm{EM}$ & $3 / 3,5$ & 1,5 & 0,9 & 2,3 & 12 & 2 \\
\hline 10 & Rohrhahngund & 56 & 2 & & 4 & 4 & II & 6,4 & 5,8 & $E M$ & $25 / 3,5$ & 2,3 & 1.2 & 3,7 & 6 & 7 \\
\hline 16 & Krummer See/Melzow & 64 & 2 & & 4 & 4 & II & $\frac{42}{3}$ & $\frac{5,9}{6,9}$ & $\mathrm{EM}$ & $3,4 / 3,9$ & 1,8 & 1,2 & 2,5 & 5 & 4,2 \\
\hline 15 & Wildbergsec & $\frac{4}{32}$ & & & $\frac{4}{2}$ & $\frac{7}{2}$ & II & $\frac{3}{1.2}$ & 3,8 & & & 2 & 1,2 & 2,8 & 4 & 1,8 \\
\hline 37 & Brackensee & 26 & 7 & & 2 & $\frac{2}{2}$ & il & 6.4 & 4,2 & $\overline{E M}$ & $1,7 / 2,7$ & 1,2 & 0,9 & 1,5 & 7 & 0.7 \\
\hline 26 & Liptowsed & $-\frac{20}{55}$ & $\frac{1}{2}$ & & $\frac{2}{3}$ & $\frac{2}{3}$ & a & $\frac{0.4}{4.5}$ & $\frac{4,2}{1,1}$ & $\frac{12}{\mathrm{e}}$ & Grund & 0,5 & 0.3 & 0.7 & 3 & 0.2 \\
\hline 36 & Euckowsee & 10 & 2 & & 3 & 3 & II & 4,6 & 7 & $\mathrm{EM}$ & $2,9 / 3,8$ & 2,1 & 1.6 & 2,5 & 7 & 1 \\
\hline 12 & Kespersee & 14 & 3 & & 4 & 4 & It & 6.6 & 6.1 & $\mathrm{EM}$ & $3,5 / 4,1$ & 1,5 & 0,5 & 2 & 12 & 4 \\
\hline 47 & Erugsee & 13 & & & 3 & 1 & II & $\frac{0.0}{7.7}$ & 3,2 & $E$ & Grund & 2,4 & 1,6 & 3,1 & 5 & 3 \\
\hline 1 & Messingsee & 3 & 1 & & 1 & 1 & I & 1,2 & 4,9 & $\mathrm{EM}$ & $2,5 / 3,4$ & 1,8 & 1,3 & 2,3 & 5 & 9.4 \\
\hline 2 & Krummer See/B. & 4 & 0 & & 3 & $\frac{2}{3}$ & 1 & $\frac{2,2}{3,5}$ & 7,5 & $\mathrm{EMH}$ & $4,5 / 5,3$ & $\frac{x}{2,4}$ & 2,2 & 2.7 & 5 & 5.6 \\
\hline 3 & KJeiner Dollinsee & 7 & 4 & 3 & 3 & 3 & I & 1,4 & 3 & $\mathrm{E}$ & Grund & 1,2 & 0,5 & 2 & 7 & 5 \\
\hline 5 & Grober Rathsburgsee & 6 & 4 & - & 2 & 2 & I & 6,8 & 9 & $\mathrm{EMH}$ & $2,5 / 4,2$ & 2,4 & 1,8 & 3,3 & 6 & 7 \\
\hline 18 & Langes Bruch & 5 & 1 & - & 2 & 2 & I & 1 & 2 & $\mathrm{E}$ & Grund & 1 & 0,0 & 1.15 & 3 & 5,4 \\
\hline 42 & Krimmer See/L. & 4 & $\frac{1}{1}$ & - & $\frac{5}{2}$ & $\frac{2}{2}$ & $\frac{1}{I}$ & 3 & 1,1 & $\vec{E}$ & Grund & 1 & 1 & 1 & 2 & 0,8 \\
\hline 8 & Großer Borgsee & 2 & 1 & & 3 & 2 & I & 3 & 2,4 & $\mathrm{E}$ & Grund & 2,4 & 1,3 & Grund & 8 & 7,6 \\
\hline 7 & Kleiner Borgsee & 7 & 1 & 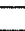 & 2 & 2 & I & 2,2 & 4,5 & $\mathrm{EM}$ & $1,9 / 2,7$ & 1,6 & 1,2 & 2,3 & 4 & 7 \\
\hline 4 & Großer Dollinsee & 1 & 1 & - & 4 & 3 & $\frac{1}{1}$ & 5,3 & 8.6 & $E M H$ & $3,8 / 5.2$ & 2.7 & 1,4 & 4,2 & 7 & 6 \\
\hline 25 & Glambecker Mühlteich & 1 & 3 & - & 2 & 2 & I & 6.8 & 1.5 & $\mathrm{E}$ & Grund & 0,6 & & & 1 & 9 \\
\hline 22 & Jacknitzsee & lLarve & & & 2 & 1 & 1 & 1.5 & 6 & $\mathrm{EMH}$ & $2,6 / 3,3$ & 1,3 & 0,7 & 1,6 & 5 & 8 \\
\hline 44 & Earschpofuhl & 2 & 1 & & 3 & 3 & 1 & 0,5 & 3,7 & & & 1 & 0,7 & 1.1 & 3 & 3 \\
\hline 17 & Aschet & $\frac{2}{1}$ & . & & $\frac{1}{1}$ & $\frac{7}{1}$ & I & $\frac{0,5}{1,7}$ & 39 & & & 2,4 & & & 1 & 5 \\
\hline 11 & Mittelsee & 1 & & & 2 & 1 & I & 7 & 4,5 & $E M$ & $3,0 / 3,7$ & 2,4 & 2 & 3 & 4 & 8,8 \\
\hline 39 & Kleiner Kagelpfuhl & 7 & 1 & - & 2 & 2 & I & 0,5 & 3 & & & 2,1 & 1,8 & 2,5 & 3 & \\
\hline 24 & Hechtdiebel & $\frac{1}{7}$ & 1 & & $\frac{2}{6}$ & $\frac{2}{2}$ & $\frac{2}{I}$ & $\frac{0,7}{1,4}$ & $\frac{3}{2,2}$ & $\mathrm{E}$ & Grund & 0,9 & 0.5 & 1.5 & 21 & 0.2 \\
\hline 34 & Schwayzer See/Blocksberg & ] & 1 & & 2 & 2 & I & 3.4 & 4,6 & $\mathrm{EM}$ & $1.9 / 2.7$ & 1,4 & 1 & 1,9 & 5 & 0,9 \\
\hline 14 & Grober Dolgensee & ] & & & 1 & 1 & $\bar{I}$ & 7 & 6 & $\mathrm{EM}$ & $2,6 / 3,6$ & 1,8 & 1,5 & 2,1 & 4 & 7 \\
\hline 45 & Paddenpfuhl & 1 & 1 & 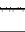 & $\frac{1}{3}$ & 2 & I & 1,3 & 5,1 & $E M_{1}$ & $2,2 / 3,2$ & 2 & 1,3 & 2,4 & 8 & 0.4 \\
\hline 38 & Moossee & $\frac{1}{5}$ & 1 & $\mathrm{t}$ & 每 & $\frac{4}{1}$ & $\frac{1}{I}$ & $\frac{1,3}{2,6}$ & $\frac{5,1}{1,3}$ & $\frac{\mathrm{E}}{\mathrm{E}}$ & Gnund & $\frac{2}{1}$ & 0.8 & 1,2 & 4 & 0.7 \\
\hline 40 & West-Rarangsee & 4 & 1 & & 7 & 2 & I & 3 & 2 & $\mathrm{E}$ & Grund & 2,2 & Gruend & Grund & 7 & 4 \\
\hline 48 & Schwarzer See/hiepe & 5 & 1 & - & 2 & 1 & I & 1,2 & 3 & $\mathrm{E}$ & Grund & 1,1 & ! & 1,4 & 3 & 5 \\
\hline 29 & Warnitzsee & 1 & 3 & 1 & 5 & 2 & I & 9 & 9,5 & $\mathrm{EMH}$ & $4 / 4,8$ & 2,3 & 0,6 & 3 & 26 & 4,5 \\
\hline 35 & Grofler Dabersee & 2 & 5 & 22 & 2 & 2 & I & 12,6 & 3,5 & $\mathrm{E}$ & $2,6 /$ & 1.3 & 0,8 & 1.8 & 6 & 2 \\
\hline 21 & Jakobsdorfer See & 1 & 3 & 1 & 3 & 3 & I & 23,5 & 10.5 & EMH & $5,2 / 5,9$ & 3,1 & 1,7 & 4,4 & 6 & 5,2 \\
\hline 23 & Laagensee & 2 & 5 & 6 & 2 & 2 & I & 35 & 4,2 & $\mathrm{E}$ & & 0,9 & 0,7 & 1,3 & 14 & 7.4 \\
\hline 43 & Kleiner Plagesee & 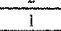 & 1 & 1 & $\frac{2}{2}$ & $\frac{2}{2}$ & $\frac{1}{1}$ & 16 & 1,6 & $\frac{2}{E}$ & Grund & 1,2 & 0.8 & 1,5 & 4 & 7.8 \\
\hline 13 & Entengritzensee & 0 & 1 & - & $\frac{2}{1}$ & 7 & + & 1,2 & 3.2 & & $?$ & 1.4 & 1 & 2 & 4 & 10 \\
\hline 19 & Kleiner Aalgastsee & 0 & 2 & . & 2 & 2 & + & 0.8 & 3.8 & $E$ & Grund & 2,2 & 1,8 & 3,1 & 6 & 12,6 \\
\hline 9 & Ochsenwerdersee & 0 & 2 & - & 1 & 1 & + & 0,6 & 1,5 & $E$ & Grund & 1,8 & - & - & 1 & 7 \\
\hline 41 & Kleiner Vätersee & 0 & 1 & 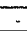 & 4 & 2 & + & 12,6 & 11,5 & $\mathrm{EMH}$ & $5,1 / 6,6$ & 3.9 & 2,6 & 5,5 & 22 & 4 \\
\hline 20 & Grober Aalgastsee & 0 & 1 & - & 1 & $\frac{6}{1}$ & + & $\frac{2,0}{2,3}$ & $\frac{112}{2,8}$ & $E$ & Grund & 0,7 & 0,4 & 0.9 & 3 & 15 \\
\hline 28 & Behlensee & 0 & 1 & $\therefore$ & 2 & 1 & + & 13.4 & 1,6 & $\mathrm{E}$ & Grund & 2,0 & Grund & $=$ & 3 & 4 \\
\hline 46 & Großer Lindsee & 0 & 1 & - & 2 & 1 & + & 1,6 & 5 & $\mathrm{EM}$ & $2,0 / 3,2$ & 1,5 & 0,6 & 2,5 & 10 & 1,4 \\
\hline 27 & Redernswalder See & 0 & 2 & 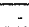 & 3 & 2 & + & 48 & 12 & EMH & $4,3 / 5,6$ & 3.1 & 2.1 & 4 & 6 & 3 \\
\hline 31 & Briesensee & 0 & 1 & - & 2 & $\mathrm{j}$ & + & 62 & 4 & $\mathrm{E}$ & Grund & 1,2 & 0.5 & 3,2 & 29 & 4.5 \\
\hline 30 & Krummer See/Neuhaus & 0 & 1 & - & 1 & l & + & 0,4 & 1,4 & $E$ & Grund & 1,6 & Grund & torund & 3 & 5 \\
\hline 49 & Máckersee & i] & l & 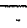 & 1 & 1 & + & 8 & 8 & $E M$ & Grund & 1,3 & - & - & 1 & \\
\hline 33 & Weiher be: Lulsenhof & 0 & 1 & 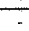 & $\frac{1}{t}$ & 1 & 4 & $\frac{0}{1}$ & 1.8 & $\overline{\mathrm{E}}$ & Grund & 2,3 & Grund & Grund & 2 & 2 \\
\hline 32 & Kiensee & 0 & 1 & 0 & 3 & 1 & + & 5.4 & 43 & $\mathrm{E}$ & & 1,5 & 0,7 & 4,3 & 40 & 6 \\
\hline A & Altwasser Rehlingen & 183 & 15 & 23 & 9 & $\frac{1}{9}$ & II & 3,7 & 5,6 & $\mathrm{E}$ & $1,9 / 2,6$ & 1,5 & 1 & 2,1 & 7 & 11 \\
\hline $\mathrm{B}$ & Oberer Altarm Beckingen & 352 & 25 & 19 & 7 & 7 & [I & 1,3 & 3,2 & $\overline{\mathrm{E}}$ & $0,0 / 1,9$ & 0,9 & 0.5 & 1.4 & 7 & 7 \\
\hline$E$ & Kieswether Ortslage Rehlingen & 93 & 8 & 29 & 7 & 6 & II & 1,8 & 3 & $\mathrm{E}$ & $1.2 / 2,2$ & 2 & 1,5 & 2,5 & 4 & 5 \\
\hline Gn & Dillinger Saar-See (bis 1993) & 42 & 4 & 20 & 7 & 6 & I & $20(3,9)$ & $\frac{3}{4}$ & $\mathrm{E}$ & $1,4 / 2,3$ & 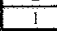 & 0,5 & 1,8 & 5 & 7 \\
\hline$c$ & Kiesweiher ASV Rehlingen & 3 & 3 & $\frac{20}{0}$ & 7 & $\frac{1}{6}$ & $\frac{a}{I}$ & $\frac{11,4}{21,4}$ & 2,1 & $\frac{L}{E}$ & $0,8 / 1,6$ & 0,8 & 0,5 & 1,2 & 5 & 6 \\
\hline $\mathrm{D}$ & A larm Pachten & $1 / /$ *** & 3 & 0 & 7 & 5 & $\mathrm{I} / * *$ & 1.5 & 4 & $\mathrm{E}$ & $7.4 / 2.2$ & 1.5 & 1.1 & 19 & 4 & 16 \\
\hline$F$ & Xiesweiher Hüttersdor 1 & 1 & 0 & 0 & 6 & 1 & $\mathrm{I}$ & 0.7 & 2,1 & $\mathrm{E}$ & $1.1 / 1.8$ & 0,8 & 0,7 & 1,1 & 4 & 5 \\
\hline F2 & Kicswieiher Hütterdorf 2 & 3 & 1 & 0 & 6 & I & 1 & 1.5 & 2,3 & $\bar{E}$ & $1,2 / 2,1$ & 0,9 & 0,6 & 1,1 & 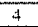 & 3 \\
\hline $\mathrm{H}$ & Kiesweiher Hector & 1 & 2 & 1 & 6 & 4 & 1 & 2,6 & 2,7 & $\mathrm{E}$ & $1,3 / 1,9$ & 0,9 & 0,6 & 1,1 & 5 & 5 \\
\hline$N$ & Kiesweiher Eisenbahnbricke & $2 /]^{* *}$ & 1 & 0 & 6 & 2 & $\mathrm{I}^{* *}$ & 2,4 & 4,7 & $\bar{E}$ & $2,6 / 3,6$ & 2 & 1.8 & 2.6 & 4 & 11 \\
\hline $\mathrm{k}$ & Fisctiteich Weserweiler & 1 & 1 & 0 & 5 & 2 & 1 & 0,8 & 2,4 & $E$ & $1,4 / 4,9$ & 1,1 & 1 & 1,3 & 4 & 2 \\
\hline 1 & Kiesteich Kreswerk I & 1 & 1 & 0 & 5 & 3 & 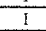 & 1 & $\frac{7}{2}$ & $\mathrm{E}$ & $1,1 * 4,6$ & 0,8 & $\frac{1}{0,7}$ & 0,9 & 4 & 5 \\
\hline 0 & Fischterch ASV Dillingen & 2 & 4 & 0 & 5 & 3 & I & 3,7 & 2 & $\bar{E}$ & $0,5 / 1,5$ & 0,5 & 0.3 & 0.7 & 4 & 8 \\
\hline $\mathrm{Rl}$ & Schwemlingen : & 2 & 0 & 0 & 2 & 2 & I & 0,15 & 2,7 & $\mathrm{E}$ & $1,3 / 23$ & 1.4 & $i_{2}$ & 1,6 & 3 & 5 \\
\hline I & Unterer Altarm Beckingen 2 & 0 & ] & 0 & 7 & 1 & + & 0,5 & 1,2 & $\mathrm{E}$ & $0,3 * 4,1$ & 0,7 & 0,6 & 0,9 & 4 & 10 \\
\hline$J$ & Fischteich ASV Büschfeld & $0 / 1 * *$ & 3 & 0 & 6 & $\overline{I(2)}$ & $+/ I^{* *}$ & 1.5 & 2 & $E$ & $0,7 / 1,6$ & 0.6 & 0,4 & 0,8 & 4 & 4 \\
\hline M) & Kesweiher Nenrig & 0 & ] & 0 & 4 & $\frac{1}{1}$ & + & 0,16 & 3,5 & $E$ & $1,4 / 2.2$ & 1,8 & 1.5 & 2,1 & 4 & 10 \\
\hline $\mathrm{P}$ & Fischteich ASV Honzrath & 0 & 7 & 0 & $\frac{\pi}{3}$ & 2 & + & $\frac{0,10}{1,7}$ & 1,7 & $\mathrm{E}$ & $0,8 / 1,3$ & 0,5 & 0,4 & 0,8 & 5 & 8 \\
\hline 0 & Altarm Rehlingen $2+3$ & 0 & $\frac{1}{2}$ & $\frac{v}{0}$ & $\frac{3}{3}$ & $\frac{2}{1}$ & + & $\frac{1.1}{0,5}$ & $\frac{1,1}{5,2}$ & $\frac{E}{E}$ & $0,5 / 3,3$ & 0,7 & 0,6 & $\frac{0,0}{0,9}$ & 5 & -3 \\
\hline
\end{tabular}


Zur Kennzeichnung der Temperaturverhältnisse (vertikale Temperaturschichtung in der Sommerstagnation) wurde nach Maßgabe der erhaltenen Profile folgende Einteilung vorgenommen:

$\mathbf{E}=$ Epilimnion (erwärmte Oberflächenschicht), $\mathbf{M}=$ Metalimnion (Sprungschicht: enormer Temperaturgradient) vorhanden, $\mathbf{H}=$ Hypolimnion (kalte Tiefenschicht) vorhanden

Als Parameter für die Sauerstoffversorgung wurde die Tiefe mit $2 \mathrm{mg} / \mathrm{l} \mathrm{O} 2$ sowie der Beginn der Sauerstoffsprungschicht aus den Profilen abgeleitet.

\subsubsection{Erklärungen für Tabelle 11}

Während Tabelle 11 die Angaben zu den Strukturen und zu der Begleitfauna klassifiziert wiedergibt, enthält Tabelle 10 dazu die Erklärung der Klassen.

\section{* Strukturen:}

Tab. 10: Häufigkeitsklassen für das Vorhandensein von Strukturen.

\begin{tabular}{|c|c|c|c|c|c|}
\hline \multicolumn{6}{|c|}{ Häufigkeitsklassen } \\
\hline Struktur & 0 & 1 & 2 & 3 & 4 \\
\hline $\begin{array}{l}\text { Schwimm- } \\
\text { blattrasen }\end{array}$ & fehlt & $\begin{array}{l}\text { nur Einzel- } \\
\text { pflanzen vor- } \\
\text { handen }\end{array}$ & $\begin{array}{l}\text { ein kleinflächi- } \\
\text { ger Bestand } \\
\text { oder mehrere } \\
\text { Einzelpflanzen } \\
\end{array}$ & $\begin{array}{l}\text { mehrere kleinflä- } \\
\text { chige oder ein } \\
\text { größerer Bestand }\end{array}$ & $\begin{array}{l}\text { geschlossener } \\
\text { Gürtel /gesamte } \\
\text { Wasserfläche } \\
\text { einnehmend } \\
\end{array}$ \\
\hline Submers, oben & fehlt & $\begin{array}{l}\text { in Einzelexem- } \\
\text { plaren vorhan- } \\
\text { den }\end{array}$ & kleinflächig & $\begin{array}{l}\text { ausgedehnte, lok- } \\
\text { ker verteilte Be- } \\
\text { stände }\end{array}$ & $\begin{array}{l}\text { großflächige, } \\
\text { dichte Bestände }\end{array}$ \\
\hline $\begin{array}{l}\text { Submers, } \\
\text { Tiefe }\end{array}$ & fehlt & $\begin{array}{l}\text { in Einzelexem- } \\
\text { plaren vorhan- } \\
\text { den }\end{array}$ & kleinflächig & $\begin{array}{l}\text { ausgedehnte, lok- } \\
\text { ker verteilte Be- } \\
\text { stände }\end{array}$ & $\begin{array}{l}\text { großflächige, } \\
\text { dichte Bestände }\end{array}$ \\
\hline Röhricht/Ried & fehlt & wenige Halme & $\begin{array}{l}\text { licht und } \\
\text { kleinflächig }\end{array}$ & schmaler Gürtel & breiter Gürtel \\
\hline $\begin{array}{l}\text { Schwingkan- } \\
\text { tenried }\end{array}$ & fehlt & $\begin{array}{l}\text { kleinflächig } \\
\text { (wenige } \mathrm{m}^{2} \text { ) }\end{array}$ & $\begin{array}{l}\text { Teil einer } \\
\text { Uferseite ein- } \\
\text { nehmend }\end{array}$ & $\begin{array}{l}\text { eine oder mehre- } \\
\text { re Uferseiten ein- } \\
\text { nehmend }\end{array}$ & $\begin{array}{l}\text { an allen Ufer- } \\
\text { seiten }\end{array}$ \\
\hline $\begin{array}{l}\text { Totholz, ober- } \\
\text { flächennah }\end{array}$ & fehlt & $\begin{array}{l}\text { wenige Äste } \\
\text { und Zweige }\end{array}$ & $\begin{array}{l}\text { verbreitet } \\
\text { Äste, Stämme, } \\
\text { Zweige }\end{array}$ & $\begin{array}{l}\text { entlang der ge- } \\
\text { samten Uferlinie }\end{array}$ & \\
\hline $\begin{array}{l}\text { Sedimente, } \\
\text { ufernah }\end{array}$ & $\begin{array}{l}\text { kahler Mine- } \\
\text { ralboden } \\
\text { (Lehm, Sand, } \\
\text { Kies) }\end{array}$ & $\begin{array}{l}\text { Mineralboden } \\
\text { mit dünner } \\
\text { Grobdetritus- } \\
\text { auflage (mit } \\
\text { Laub usw.) } \\
\end{array}$ & $\begin{array}{l}\text { Unterwasser- } \\
\text { torf }\end{array}$ & weiche Mudden & \\
\hline Ufergehölze & fehlen & $\begin{array}{l}\text { vereinzelt }(10 \% \\
\text { der Uferlänge) }\end{array}$ & $\begin{array}{l}<50 \% \text { der } \\
\text { Uferlänge }\end{array}$ & $\begin{array}{l}>50 \% \text { der Ufer- } \\
\text { länge }\end{array}$ & $\begin{array}{l}\text { geschlossener } \\
\text { Saum }(>90 \%) \\
\text { der Uferlänge }\end{array}$ \\
\hline
\end{tabular}




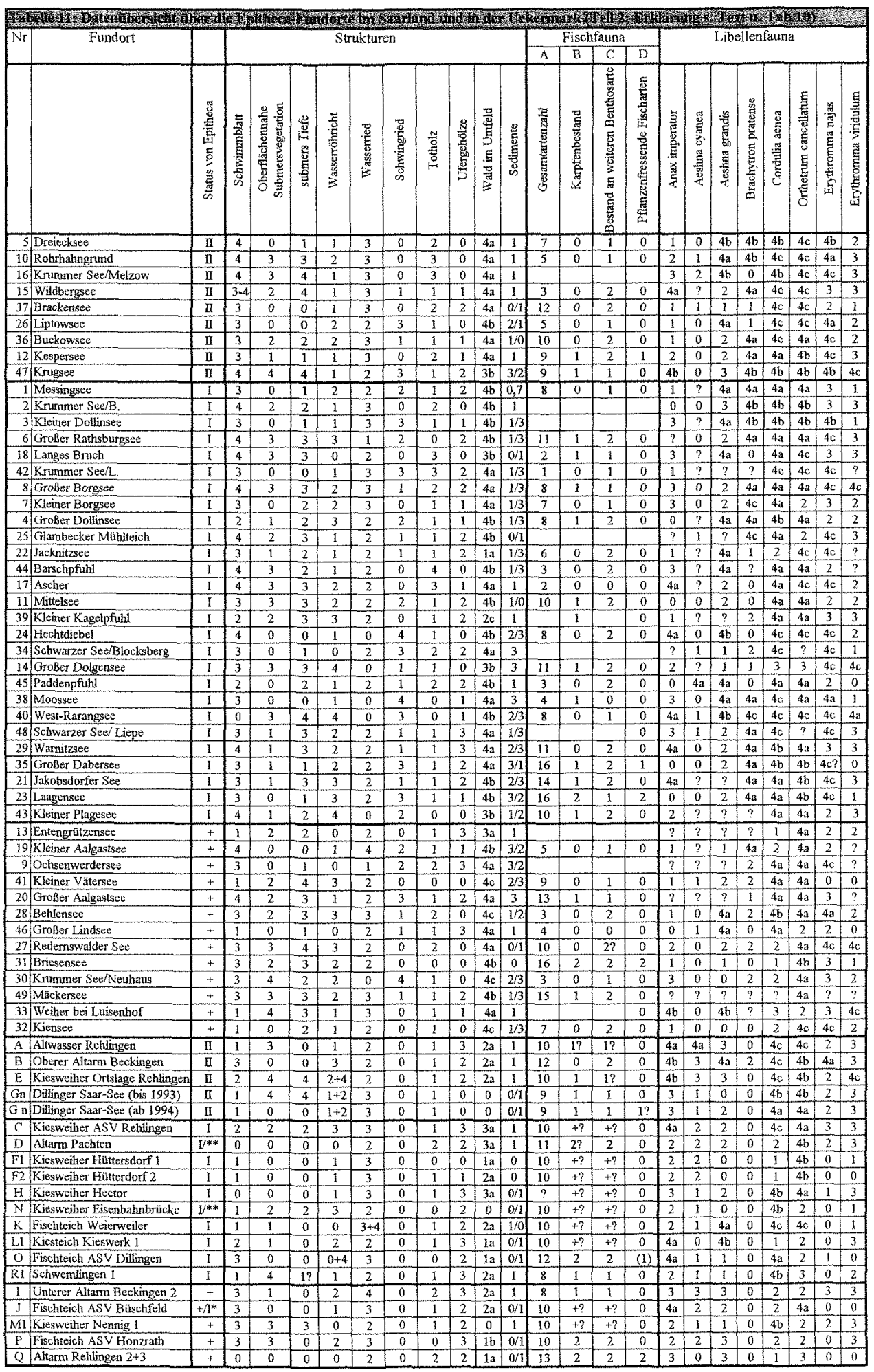


Eine Auswertung der Wald- bzw. der Gehölzbestände in der Umgebung des Gewässers (geschlossene Gehölzbestände größer 10 ar) wurde durch folgende Einteilung vorgenommen:

$0 \quad$ kein Wald im Umfeld von $100 \mathrm{~m}$ um das Gewässer

1 Gehölzbestand oder Wald im Umfeld von $100 \mathrm{~m}$ kleinflächig vorhanden

2 Wald an höchstens $50 \%$ der Uferlinie angrenzend

3 angrenzender Wald an mehr als 50\% des Ufers

4 Gewässer vollständig von Wald umgeben

Die Art der Bestockung wurde dabei zusätzlich durch folgende drei Kategorien erfaßt:

$\begin{array}{ll}\text { a } & \text { Laubwald } \\ \text { b } & \text { Laub-Nadel-Mischwald (oder nebeneinander Laub- und Nadelholzbestände) } \\ \text { c } & \text { Nadelwald }\end{array}$

\section{* Fischfauna:}

Für eine kurze Kennzeichnung der Fischfauna wurden als relevante Parameter ausgewählt:
A: $\quad$ Artenzahl
B: $\quad$ Karpfen (Cyprinus carpio)
C: $\quad$ weitere Grundfische: Blei (Abramis brama), ferner auch Güster (Blicca bjoerkna), Karausche (Carassius carassius) und Schlei (Tinca tinca)
D: Pflanzenfressende, fremdländische Fischarten: Graskarpfen (Ctenopharyngodon idella),
Silberkarpfen (Hypophtalmichthys molitrix) und Marmorkarpfen (Aristichthys nobilis)

Sofern möglich, wurde folgende Einteilung für die Bestandsangaben für $\mathrm{B}, \mathrm{C}$ und $\mathrm{D}$ benutzt:

$\begin{array}{ll}0 & \text { fehlt } \\ 1 & \text { in wenigen Exemplaren } \\ 2 & \text { größerer Bestand }\end{array}$

\section{* Libellenfauna:}

Die Einordnung des Status der 8 ausgewählten Arten erfolgte nach Abundanzen und dem Grad der Indigenität:

$\begin{array}{llll}\ldots ? & \text { unzureichende Bearbeitung } & \ldots 3 & \text { Eiablagen/Kopula } \\ \ldots 0 & \text { nicht nachgewiesen } & \ldots 4 \mathrm{a} & \text { Exuvien/Larven } \\ \ldots 1 & \text { Einzelfunde } & \ldots 4 \mathrm{~b} & \text { bodenständig (mind. 2 Jahre 4a) } \\ \ldots 2 & \text { Imagines an mehreren Tagen } & \ldots 4 \mathrm{c} & \text { bodenständig in hohen Abundanzen }\end{array}$

\section{Diskussion}

Epitheca bimaculata gilt als sehr selten und in vielen Teilen Mitteleuropas sogar als verschollen. Zum anderen häufen sich in den letzten Jahren Fundmeldungen aus Teilen Deutschlands und Frankreichs (Zusammenstellung z. B. in HESS u. a., 1996, KITT \& WOLF, 1995, REDER, 1992, TROCKUR \& DIDION, 1994, MAUERSBERGER \& MAUERSBERGER, 1992, L.E.G.- 
U.A.N., 1993, Verbreitungskarte bei DOMMANGET, 1994 bzw. Erwähnung in HEIDEMANN \& SEIDENBUSCH, 1993). Zweifellos ist ein hoher Anteil der neuen Funde auf intensivere Untersuchungsaktivität zurückzuführen. Mit einer Stetigkeit von $22 \%$ an den Seen des uckermärkischen Untersuchungsgebietes (MAUERSBERGER \& MAUERSBERGER, 1996) kann Epitheca hier nicht mehr zu den seltenen Arten gerechnet werden.

HESS u. a. (1996) interpretieren im Zusammenhang mit der Diskussion zu den alten und aktuellen Funden in Bayern die zeitlichen und räumlichen Häufungen als Ausbreitungswellen mit einer meist kurzfristigen Ausbildung lokaler Metapopulationen. Wir vermuten, daß die eigentlichen Stammhabitate der Art in Gebieten mit Nachweisen noch nicht entdeckt sind eine gezielte systematische Durchsuchung wie im Saarland oder in der Uckermark fehlt in Bayern bisher. Mit großer Wahrscheinlichkeit handelt es sich bei dem über Jahre mit Imaginalnachweisen belegten Vorkommen an den beiden oberschwäbischen Urseen um solche Stammhabitate. An den Stammgewässern unserer Studie wurde eine kontinuierliche Besiedlung über mehrere Jahre nachgewiesen.

Folgende Gründe könnten erklären, warum diese große Libelle bislang vielerorts übersehen wurde:

- die Imagines sind farblich unauffällig, die Männchen patrouillieren oft weitab vom Ufer über größeren Wasserflächen und werden somit übersehen oder verwechselt,

- Epitheca-Imagines gehören zu den leistungsfähigsten Flugorganismen, setzen sich selten im Uferbereich erreichbar ab und werden daher auch nur ausnahmsweise gefangen,

- die Gewässertypen mit individuenreichen Epitheca-Brutkolonien sind odonatologisch ansonsten oft wenig ergiebig und wurden kaum (vollständig) untersucht,

- die Art hat eine sehr kurze Emergenz- und Flugperiode.

Allerdings sind die Exuvien sehr auffällig, nicht zu verwechseln und leicht zu finden, sofern gezielt danach gesucht wird.

\subsection{Morphometrische Kennzeichnung der Epitheca-Gewässer}

Die Wassertiefe besitzt für die Imagines keinerlei direkte Relevanz, da sie gerade wegen der Trübung der Gewässer in keiner Weise wahrgenommen werden kann. Für die Larvenstadien dürfte die Wassertiefe aber aus mehreren Gründen enorme Bedeutung haben.

Das tiefste Epitheca-Gewässer im Saarland (Altwasser Rehlingen) erreicht knapp 6 Meter Tiefe, was aber weniger den Habitatanspruch der Libellenart kennzeichnet, sondern die Gewässersituation des Gebietes - im Umkreis von mehr als $20 \mathrm{~km}$ gibt es kein tieferes Gewässer. In der Uckermark wurde Epitheca ausnahmsweise auch an Seen mit Maximaltiefen von 10,5 bis 12 Metern nachgewiesen, bei denen aber vor allem flachere Buchten für die Art von Bedeutung sind. Tiefere Seen werden von Epitheca offenbar gemieden.

Der Larvenlebensraum befindet sich im Sommer vermutlich nie unter etwa 2,5 m Tiefe, was aus den Sauerstoffdefiziten und der niedrigen Wassertemperaturen in der Tiefe der meisten Gewässer zu schließen ist - und daraus, daß auch Gewässer besiedelt werden, die nirgends diese Tiefe erreichen. So genügen im Saarland für eine erfolgreiche Reproduktion maximale Tiefen von gut 2 Metern, die flachsten Epitheca-Gewässer in der Uckermark erreichen gerade einmal 1,1 m (Liptowsee) und 1,3 m (Moossee). Damit ist immer noch ausreichender Schutz gegen Durchfrierung und die Möglichkeit der Besiedlung durch Fische (s. 4.7.1) gewährleistet. 
Das flachste uns bekannte stetige Brutgewässer der Art ist der Schwarze See im Göldenitzer Moor (MAUERSBERGER \& WAGNER, 1990) mit 0,7 Meter Maximaltiefe, der allerdings durch seine Nähe zur Ostsee in einem wintermilden Klimabereich liegt.

Epitheca bimaculata gilt als typische Seen-Libelle (z. B. BELLMANN, 1987, JURZITZA, 1988, JAHN, 1982, SCHMIDT, 1975, DREYER, 1986, MÜNCHBERG, 1932, FRÖHLICH, 1900). Wir konnten nachweisen, daß $85 \%$ der besiedelten Gewässer kleiner als 8 Hektar sind, viele davon allerdings liegen von der Fläche her noch unterhalb oder an der Grenze gängiger Seedefinitionen.

\section{2. Über die geringe Bedeutung der Wasserbeschaffenheit für Epitheca}

Aus Tabelle 9 läßt sich ableiten, daß Epitheca durchaus subneutrale (schwach saure) Weichwasserökosysteme besiedelt, daß aber der Schwerpunkt äquivalent zum Angebot in den untersuchten Landschaften bei den alkalischen Gewässern mit höherer bis hoher Wasserhärte zu suchen ist. Spitzenwerte des $\mathrm{pH}$-Wertes bis 9 , infolge starker Photosyntheseaktivität gerade bei nährstoffreicheren, hochproduktiven Seen häufig, stellen für Epitheca offenbar keine limitierende Belastung dar.

$\mathrm{Da} ß$ unter den Fortpflanzungsgewässern in der Uckermark der Anteil der Weichwasserseen höher ist, als es bei der naturräumlichen Ausstattung wahrscheinlich wäre, hat nichts mit der Bedeutung der Wasserbeschaffenheit für die Art zu tun, sondern spiegelt lediglich eine Eigenschaft der mit Vorliebe besiedelten Waldseen in der Endmoräne wieder.

Auch aus der Literatur ließen sich bereits Hinweise auf eine große Variabilität hinsichtlich der Wasserchemie erwarten (z. B. DONATH, 1987). JAQUEMIN u. a. (1985) beschreiben einen Exuvienfundort in den Vogesen als torfigen Weiher mit saurem Wasser und Sphagnen. DEGRANGE \& SEASSAU (1974) fanden Larven bei einem pH-Wert von 4,5. Derart saure Gewässer existieren zwar im uckermärkischen Untersuchungsgebiet auch, werden aber von Epitheca gemieden; die Ursachen liegen allerdings wohl in der Beschaffenheit der Sedimente (s. 4.5.3) und der fehlenden Besiedlung durch Fische (s. 4.6.1).

Die Transparenz der Gewässer unterscheidet sich zwar deutlich in Uckermark und Saarland; bei den Stammhabitaten zeigt sich allerdings erstaunliche Übereinstimmung (s. Tab. 5 in Kap. 4.4.2) - die Optimalgewässer der beiden untersuchten Regionen sind sich in diesem Parameter somit recht ähnlich. Etwas trübe (eutrophe) bis stark getrübte (polytrophe) oder braungefärbte (meso- bis euhumose) Gewässer bilden einen gewissen Schwerpunkt der Besiedlung.

Ausgesprochen klare, nährstoffarme Seen (oligo- oder mesotroph) erweisen sich als ungünstig; als Ursache kommt z. B. ein Mangel an Schwimmblattrasen oder ein schwächerer Temperaturgradient in Frage.

Im Hinblick auf den Sauerstoffhaushalt ist Epitheca bei den Gewässern nicht wählerisch. Die Larven bewegen sich in Wassertiefen, wo auch stärker belastete Gewässern stets ausreichend Sauerstoff bieten. Vorübergehendes Fehlen von Sauerstoff im Winter wirkt sich zumindest auf die großen Larven nicht schädigend aus.

Im ungewöhnlich kalten Winter 1995/96 mit fast 18 wöchiger Eisbedeckung stickten unter anderem 7 der 9 Stammhabitate in der Uckermark aus (Sauerstoff über Wochen bei $0 \mathrm{mg} / \mathrm{l}$ ). Die Emergenzrate 1996 zeigte keine Unterschiede gegenüber den Vorjahren. Auch der experimentelle Entzug von Sauerstoff führte nicht zu Verhaltensänderungen der Larven in den Aufzuchtgefäßen. 


\subsection{Die Struktur der Sedimente an Epitheca-Gewässern}

In den potentiellen Larvenlebensräumen (ufernahe Seebereiche) wurden besonders häufig mineralische Unterwasserböden mit einer dünnen Auflage aus zumeist gröberem organischen Material gefunden. Seltener kommen auch dickere Schichten organischer Sedimente (Grobdetritusmudden und subaquatische Torfe) vor.

Feindetritusmudden, die als suspendierte Planktonablagerungen der gereiften Seeökosysteme oberflächlich oft sehr weich sind, stellten sich als besiedlungsfeindlich heraus.

Die Sedimentoberfläche in Epitheca-Gewässern ist offenbar derart strukturiert, daß ein partielles Eingraben der Larven als Schutz vor Prädatoren möglich ist, aber ein Versinken und Ersticken in sauerstoffarmen Schichten vermieden werden kann. Das Schutzverhalten wird durch die seitlich abgeplattete Form des Abdomens und die extrem langen Beine unterstützt. Mit dieser Präferenz kommen Seen mit Mineralbodenufern ebenso als Fortpflanzungshabitat in Frage, wie größere Altarme in Flußauen und relativ junge Abgrabungsgewässer.

\subsection{Bedeutung der Wasservegetation}

Relativ groß ist die Anzahl von Literaturstellen, die für Epitheca-Fundstellen in erster Hinsicht reichlich Schilf- und Binsenbestände angegeben: z. B. DREYER, 1986, NIEHUIS 1984, FRÖHLICH, 1900.

Zunehmend ist die Zahl von Autoren, die in ihren Beschreibungen von Gewässern mit Epitheca-Nachweisen das Vorhandensein von Schwimmblattrasen und/oder Tauchfluren hervorheben (z. B. DUVAL \& PRATZ, 1988, JAQUEMIN u. a. 1985, JAHN, 1982, KOTARAC, pers. Mitt.). Bei DONATH (1987) werden zusätzlich Grundrasen erwähnt, die für Epitheca nicht erforderlich sind (z. B. Dreiecksee ist frei von submerser Vegetation).

Tab. 12: Anzahl der Eistränge an verschiedenen Substraten (Saarland 1992-1995).

\begin{tabular}{|l|c|c|c|c|}
\hline & $\begin{array}{c}\text { Altwasser } \\
\text { Rehlingen (A) } \\
(\mathrm{n}=25)\end{array}$ & $\begin{array}{c}\text { Oberer Altarm } \\
\text { Beckingen (B) } \\
(\mathrm{n}=41)\end{array}$ & $\begin{array}{c}\text { Kiesweiher } \\
\text { Rehlingen (E) } \\
(\mathrm{n}=46)\end{array}$ & $\begin{array}{c}\text { Saar-See } \\
\text { Dillingen }\left(\mathrm{G}_{\mathrm{n}}\right) \\
(\mathrm{n}=26)\end{array}$ \\
\hline Myriophyllum & 13 & & 46 & 3 \\
\hline Elodea & & 7 & & 23 \\
\hline Nuphar & & 13 & & \\
\hline $\begin{array}{l}\text { Phragmites } \\
\text { (Halme vom Vorjahr) }\end{array}$ & & 5 & & \\
\hline Riedstrukturen & & 16 & & \\
\hline Totholz (Äste, Zweige) & 9 & & & \\
\hline Totholz (grün, gefällt) & 3 & & & \\
\hline
\end{tabular}

Die drei bisher entdeckten individuenreichsten Brutkolonien Europas (COPPA, 1990: Loc. F, Dreiecksee/U und Oberer Altarm Beckingen/S) haben ausgedehnte Bestände der Teichrose (Nuphar lutea) gemeinsam. Dieses Habitatmerkmal findet sich in der Oberrheinebene 
(KITT, pers. Mitteilung), im Norden Weißrußlands (eigener Nachweis) wie auch in Skandinavien wieder (vgl. Foto bei SANDHALL, 1990). Oftmals tritt bei Epitheca-Gewässern noch Submers-Vegetation (z. B. Myriophyllum, Ceratophyllum) als Ergänzung oder Ersatz auf. Die Bedeutung dieser Strukturen bei der Habitatselektion wird jedoch erst richtig deutlich, wenn man sich die Substrate für die Ablage der etwa 1600 Eier zählenden Eistränge ansieht (s. Tab. 12 in Verbindung mit Tab. 8).

Die enorme Bedeutung der oberflächennahen Habitatstrukturen läßt sich anhand der Analyse der Eiablagen sowie im Falle eines Verlustes der Strukturen erkennen: die Eiablagen erfolgen oberflächennah an emerse bzw. submerse Strukturen in Abhängigkeit von deren Häufigkeit. Fehlt eine gut ausgebildete Tauchblatt- oder Schwimmblattvegetation an einem Gewässer, so wird Epitheca bimaculata dieses nicht oder nur vereinzelt und kurzfristig bzw. nicht jedes Jahr aufsuchen. In der Literatur wird dann vielfach von unregelmäßigem Auftreten oder Einzelfunden berichtet (LE ROI, 1913, JURZITZA, 1965, VINCENT, u. a. 1987).

Bestätigt wird diese Hypothese durch Gewässerveränderungen, die im Saarland am Dillinger Saar-See im Sommer 1993 stattfanden:

Innerhalb weniger Wochen verschwanden alle Elodea- und Myriophyllum-Bestände, die noch im Juni zusammen mit Watten aus fädigen Grünalgen den Aspekt des 20 ha großen, max. 3,5 m tiefen Flachsees bestimmten. Das Gewässer war im August 1993 nunmehr geprägt durch eine äußerst geringe Sichttiefe aufgrund einer „Blüte“ koloniebildender Grünalgen. Der Verlust an Wasserpflanzen ist nicht sicher erklärbar, vermutet wird ein Besatz mit Graskarpfen im Zeitraum Ende Juni-Anfang August 1993.

Die Folgen des Verlustes der Vegetationsstrukturen für Epitheca bimaculata waren in den Folgejahren überdeutlich. Im Mai 1994 schlüpften mit 43 aufgesammelten Exuvien zwar noch die höchsten Anzahlen im Untersuchungszeitraum. Trotz intensiver Untersuchungsaktivität (10 Begehungen bei günstiger Witterung in der Flugzeit) konnte jedoch kein einziges Männchen in der Flugperiode 1994 angetroffen werden, auch Eistränge wurden nicht mehr gefunden. Gleichzeitig tauchten erstmalig an zwei unmittelbar benachbarten, relativ strukturarmen Gewässern Epitheca-Männchen revierbildend auf.

Der Einbruch in der Larvenpopulation wurde erst in den nachfolgenden Jahren bei der Emergenz deutlich (man beachte die potentiell 2-3jährige Entwicklungszeit): 1995 konnten nur noch 4, 1996 gar nur noch eine Exuvie in den kontrollierten Bereichen des Nordufers angetroffen werden. Männchen-Nachweise gelangen auch 1995 und 1996 nicht mehr.

Durch die Zerstörung der Wasserpflanzen wurde der Larvallebensraum der letzten Stadien (vgl. Kap. 5.3.) nicht beeinträchtigt, wie die relativ zahlreichen Exuvienfunde an jenem Gewässer von 1994 belegen.

\subsection{Epitheca-Gewässer liegen im Laubwald}

Diese Aussage gilt in sehr vielen Fällen und vornehmlich für die uckermärkischen Stammhabitate (nach BEUTLER, mdl., aber auch für Südost-Brandenburg). Aus Figur 8 geht hervor, daß besiedelte Gewässer in den überwiegend mit Buchenalthölzern bestockten, von kleinen Erlenbruchwaldflächen durchsetzten Endmoränenzügen konzentriert sind. An Seen in der Offenlandschaft und in den Kiefernforsten der Schorfheide fehlt die Art fast gänzlich.

Im Saarland ist diese Beziehung nicht ohne weiteres erkennbar, jedoch sind auch hier an nahezu jedem Gewässer Erlen- oder Weidenufergehölze sowie Pappelgruppen oder andere Laubgehölze in der Nähe bzw. direkt am Ufer vorhanden. Ausgedehnte Wälder sind in vielen Fällen nicht weit entfernt. 


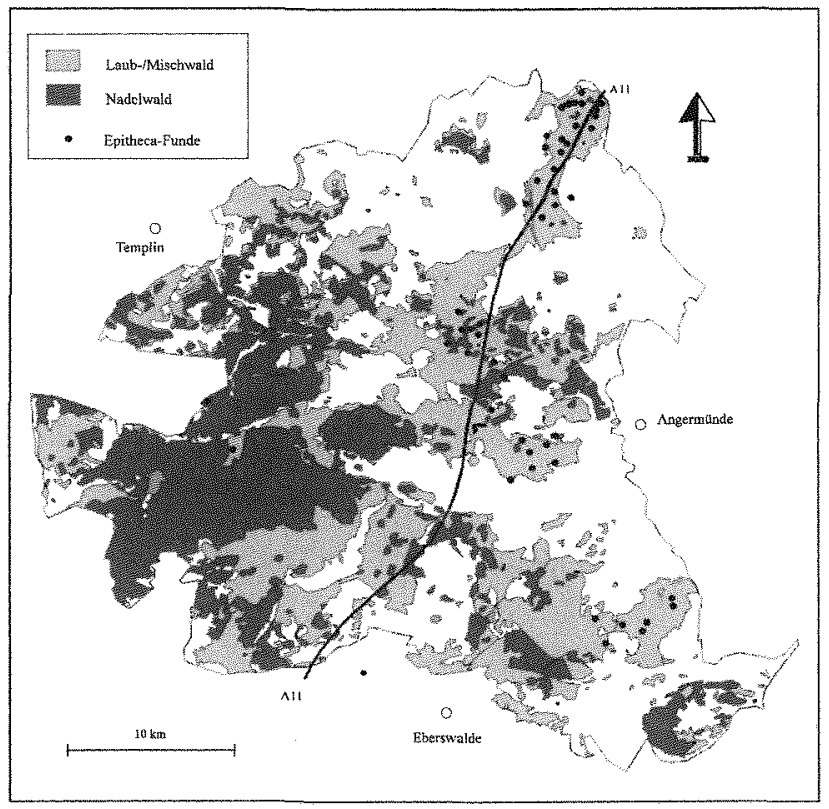

Fig. 8: Zusammenhang zwischen Vorkommen von Laubwald und Epitheca bimaculata im Biosphärenreservat „Schorfheide-Chorin“ (Uckermark).

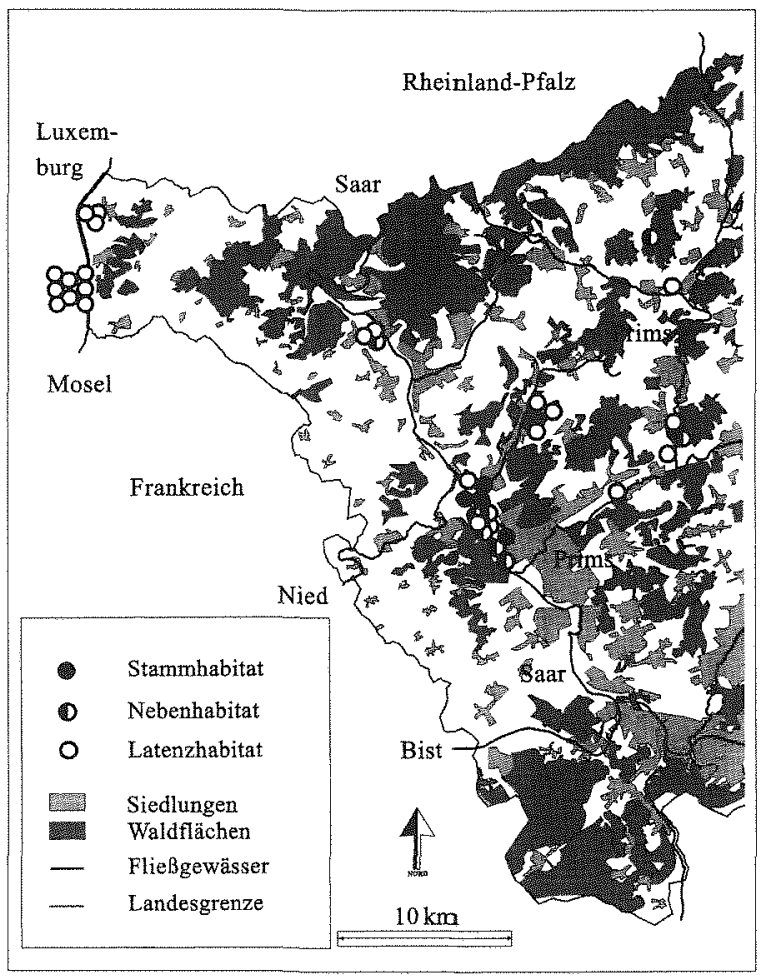

Fig. 9: Lage der saarländischluxemburgischen Epitheca-Fundorte. 
Der forstlich eingeleitete Laubwaldverlust zugunsten der Kiefern und Fichten in den jungpleistozänen Seengebieten Mecklenburgs, Brandenburgs, Baden-Württembergs und Bayerns (sowie der heutige Mangel an nicht verbauten Flußauen mit Auwäldern) könnte eine der wichtigsten Ursachen für die Gefährdung von Epitheca bimaculata sein.

Uns gelang bislang keine endgültige Klärung, worin die Bindung an die gewässernahen Laubgehölze besteht. Folgende Sachverhalte wären zu diskutieren:

- die hohen Gehölzbestände bilden eine windbremsende Kulisse bzw. ein eigenes Mikroklima für den Revierflug der Männchen über der offenen Wasserfläche,

- der Eintrag von Fallaub in die Flachwasserbereiche trägt zur Bildung der bevorzugten Sedimentbeschaffenheit als Larvenlebensraum bei,

- Gehölze bieten Unterschlupf und Schutz in der Nacht und in Schlechtwetterperioden, - Laubgehölze, vielleicht vorrangig die Kronenbereiche reich strukturierter Wälder, könnten der Imaginallebensraum während der Reifungsphase sowie der Jagdlebensraum sein.

Für den letztgenannten Aspekt sprechen eigene Beobachtungen zum Jagdflug bei schwülwarmer Witterung sowie abends oder morgens: die Tiere flogen langsam und ausdauernd sehr hoch über der Wasserfläche in Höhe der Baumkronen der umgebenden Gehölze. Nach SONEHARA (1967) jagen die Tiere der Subspezies sibirica in Japan in der etwa 10 Tage dauernden Reifungsphase meist über Bäumen.

\subsection{Anpassung gegenüber Prädatoren}

Die größte Gefährdung durch Prädatoren für Epitheca stellen offenbar Molche und Wasserinsekten (große Schwimmkäfer und deren Larven, Larven von Mosaikjungfern) dar. EpithecaLarven im vorletzten Stadium wurden in eigenen Folienteich-Freilandversuchen $(\mathrm{S}+\mathrm{U})$ von großen Dytisciden-Larven und vermutlich von Molchen gefressen und kamen nicht zur Emergenz. Die gleichfalls aktiv jagenden Aeshniden-Larven stehen ebenso im Verdacht, bedeutende Epitheca-Prädatoren zu sein: die Exuvien-Abundanz von Aeshna cyanea und Anax imperator war an den von uns untersuchten Gewässern stets gering (s. a. Tab. 11). Auch schlugen zweimalige Ansiedlungsversuche der Art an einem fischfreien, aber an Aeshniden reichen (Aeshna cyanea, Ae. juncea und Anax imperator) Waldteich (S) fehl.

Um den genannten Freßfeinden zu entgehen, besiedelt der Zweifleck womöglich ausschließlich Gewässer, die von Fischen bewohnt werden. Damit scheiden allerdings die meisten Kleingewässer und auch stark saure Moorrestkolke als Habitat aus.

Aus dieser Koexistenz mit Fischen (vgl. auch JOHNSON \& MOORE, 1991) resultieren außerdem für die Larven

- eine vorwiegend kryptische Lebensweise (im Sediment versteckt, sit-and-wait-Libelle, „slow lifestyle"),

- eine vorwiegend nächtliche Aktivität/Nahrungsaufnahme (belegt durch eigene Versuche),

- die Ausbildung stachliger Körperfortsätze (extrem lange Lateral- und Dorsaldorne am Abdomen),

- eine lange Entwicklungszeit (2 bis 4 Jahre im Laborversuch unter relativ naturnahen Bedingungen).

In Figur 10 ist der Larvenlebensraum der Art schematisch dargestellt. 


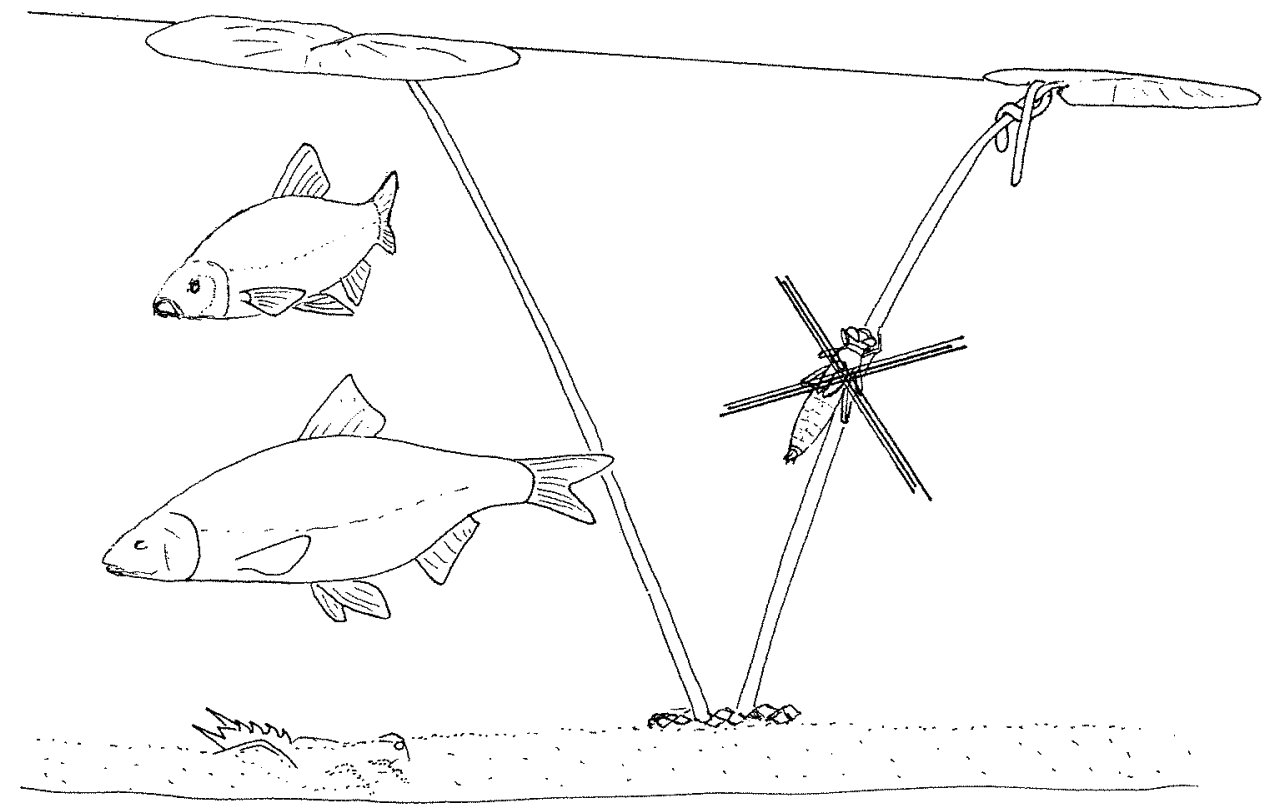

Fig. 10: Schema zu den Bedingungen im Larvalhabitat von Epitheca bimaculata.

Die üblicherweise geringe Abundanz von Epitheca könnte darauf zurückgeführt werden, daß die Prädation durch Fische trotz Anpassung hohe Verluste verursacht. Im Wechselspiel Schutz - Prädation bei der Koexistenz mit Fischen könnte auch die Ursache für die evolutive Entwicklung des in Europa bei Libellen einzigartigen Eiablageverhaltens (konzentrierte Eiablage durch Eistränge mit größenordnungsmäßig 1600 Eiern) liegen.

Die Stammhabitate mit ihrer großen Individuendichte besitzen gegenüber Nebenhabitaten tendentiell auch geringere Bestände von Fischarten, die am Gewässerboden nach Nahrung suchen (z. B. Blei, Karpfen, s.a. Tab. 11) und damit einerseits Prädator und andererseits Konkurrent für die Larven sein könnten!

\subsection{Bemerkungen zu Negativfaktoren, Optimalhabitat und typischem Land- schaftsraum}

\subsubsection{Negativfaktoren und Optimalhabitat}

In den voranstehenden Kapiteln wurden die Merkmale der Epitheca-Gewässer betrachtet und dabei zumeist die Optimallebensräume (Stammhabitate) hervorgehoben.

Parallel wurde versucht, Gewässer in den Untersuchungsgebieten ausfindig zu machen, an denen die Art im Untersuchungszeitraum definitiv nicht vorkommt. Mit letzter Sicherheit ist dieser Status zwar nicht beweisbar, jedoch konnten wir einige Libellengewässer zur Auswertung heranziehen, an denen auch nach 3 oder gar 7 Jahren Beobachtung kein Nachweis der Art gelang, obwohl unsere Methodik für deren Erfassung ausreichend ausgereift sein dürfte. 
Es handelt sich im Saarland um folgende Gewässer: Altarm Mechern, Altarm Niedmündung, Saar-See (flache Bucht), Waldteich nördl. Eisen, kleiner Fischteich Dillingen, Restgewässer 1 und 3 des unteren Altarmes bei Beckingen, Fischteich Dagstuhl, Fischteich Geisweiler Hof, kleiner Teich Hausstadt, Moselaltarm. Im Untersuchungsgebiet Uckermark sind es 6 Seen in der Schorfheide (Barssee bei Gr. Väter, Gr. Gollinsee, Krummer und Runder Köllnsee, K1. Lotzinsee, Wuckersee) sowie der Plötzendiebel bei Glambeck, der Kl. Rathsburgsee bei Meichow und der Schmale Temmensee.

Diese Gewässer wurden dem gleichen Untersuchungsprogramm wie die Epitheca-Fundorte (Tab. 9 und 11) unterzogen, um Eigenschaften herauszufinden, die die Besiedlungsmöglichkeiten für die Art begrenzen.

Von diesen Epitheca-freien Gewässern werden verschiedene der in den vorangegangenen Kapiteln als bedeutsam herausgestellten Merkmale nicht erfüllt, denn sie sind:

- zu klein (6 mal S + U), zu groß (1 mal U),

- zu flach (2 mal S), zu tief (2 mal U),

- zu trüb und belastet (1 oder 2 mal S), zu klar und nährstoffarm (3 mal U),

- zu kalkarm und damit sauer, frei von Friedfischen und reich an Aeshniden ( 3 mal $S+U$ ),

- zu arm an geeigneten emersen Strukturen (6 mal S+ U),

- ausschließlich mit zu weichen Sedimenten ausgestattet (3 mal U),

- zu arm an Ufergehölzen oder Laubwald in der Umgebung (8 mal S+U).

Pro Gewässer konnten somit 1 bis 4 ungünstige Eigenschaften erkannt werden. Nur beim Schmalen Temmensee werden alle von uns ermittelten Bedürfnisse erfüllt - und trotzdem konnte die Art nicht nachgewiesen werden.

Tab. 13: Optimalhabitat und Ausschlußkriterien von Epitheca bimaculata.

\begin{tabular}{|l|l|l|}
\hline Parameter & Optimum & Negativfaktoren \\
\hline Größe & 4 ha & kleiner als 0,5 ha, größer als 15 ha \\
\hline Tiefe, maximal & $1,5-5 \mathrm{~m}$ & flacher als 1 m, tiefer als 8 m \\
\hline Wasserbeschaffenheit & eutroph, meso- euhumos & oligo- o. hypertroph, oligohumos \\
\hline Vegetationsstrukturen & $\begin{array}{l}\text { flächenhaft ausgebildete, ober- } \\
\text { flächennahe Strukturen }\end{array}$ & $\begin{array}{l}\text { Schwimm- und Tauchblattstruktu- } \\
\text { ren fehlen völlig }\end{array}$ \\
\hline Sedimente in Ufernähe & Grobdetritus über Sand & $\begin{array}{l}\text { nackter Kies, weiche Feindetritus- } \\
\text { mudden }\end{array}$ \\
\hline Begleitfauna & $\begin{array}{l}\text { Fische sind in mehreren Arten } \\
\text { und natürlichen Abundanzver- } \\
\text { hältnissen vorhanden }\end{array}$ & $\begin{array}{l}\text { Friedfische fehlen oder Massen- } \\
\text { vorkommen von Karpfen vorhan- } \\
\text { den }\end{array}$ \\
\hline $\begin{array}{l}\text { große räuberische Wasser- } \\
\text { insekten, Molche }\end{array}$ & $\begin{array}{l}\text { fehlen oder in geringer Abun- } \\
\text { danz }\end{array}$ & $\begin{array}{l}\text { Dytisciden, Anax imperator, Ae- } \\
\text { shna cyanea, Triturus spec. in ho- } \\
\text { her Abundanz vorhanden }\end{array}$ \\
\hline Umgebung & $\begin{array}{l}\text { Ufergehölze und/oder angren- } \\
\text { zender Laubwald }\end{array}$ & \begin{tabular}{l} 
ausgeräumte Agrarlandschaft \\
\hline
\end{tabular}
\end{tabular}



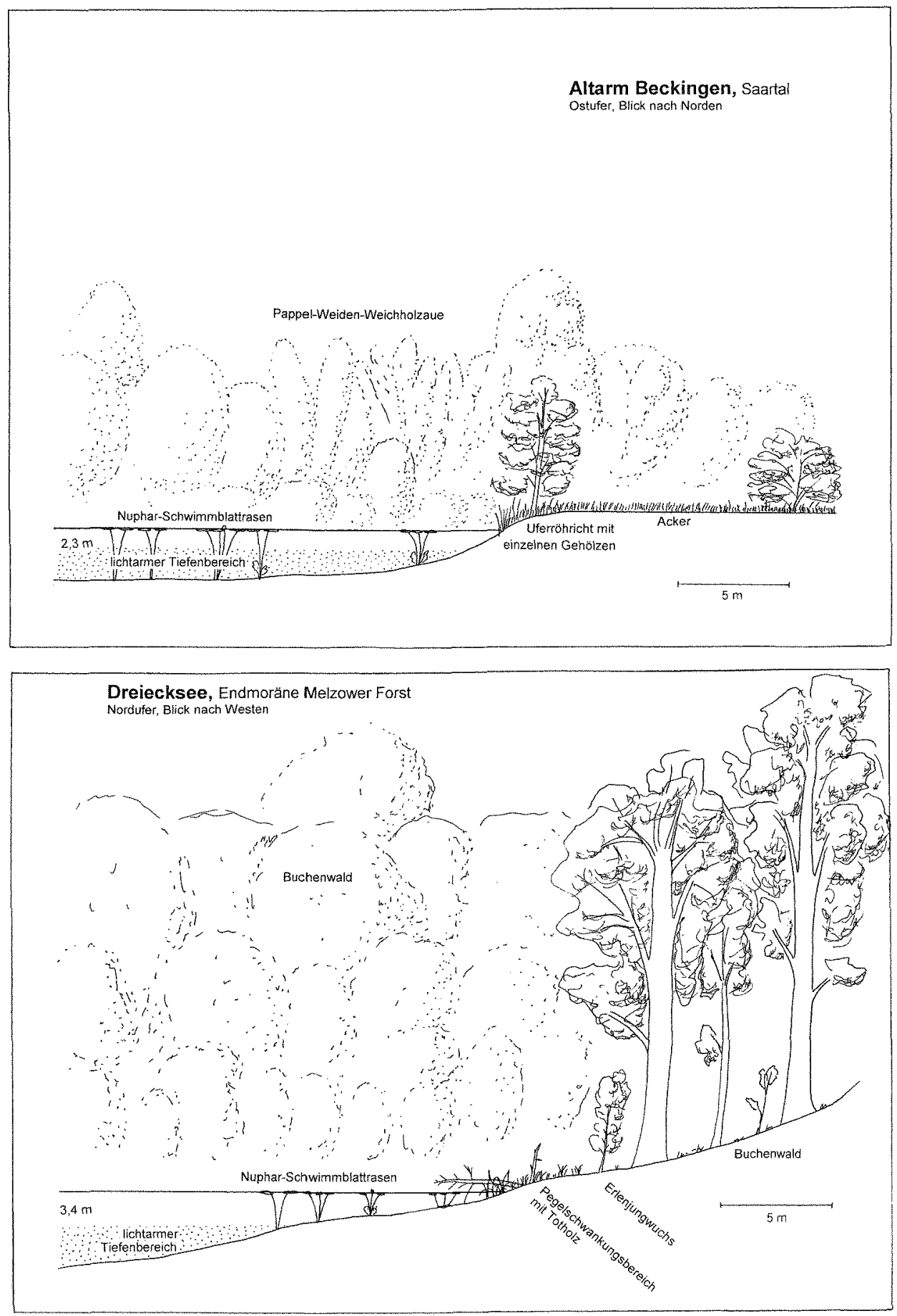

Fig. 11: Gewässer mit bis zu 400 schlüpfenden Individuen pro Jahr im Profil: Oberer Altarm Beckingen (S) und Dreiecksee (U). 
Aus den Vergleichen der besiedelten Gewässer untereinander und mit den „Negativgewässern“ läßt sich die in Tabelle 13 dargestellte Struktur eines typischen Optimalhabitates beschreiben. Zur Veranschaulichung ist in Figur 11 außerdem der Landschaftseindruck an den beiden individuenreichsten bekannten Epitheca-Gewässern Deutschlands dargestellt.

In Mitteleuropa sind zwei Landschaftstypen zu nennen, die die bevorzugten Fortpflanzungsgewässer in größerer Zahl von Natur aus aufwiesen: Flußauen und Seenlandschaften.

\subsubsection{Epitheca bimaculata in Flußauen}

Wir gehen ebenso wie SCHMIDT (1988) davon aus, daß einst Altwässer und Altarme der Flußtäler mit nicht zu kleiner Wasserfläche, umgeben von Hart- und Weichholzauwäldern einen Besiedlungsschwerpunkt der Art in Europa bildeten. Insbesondere der Nahrungsreichtum (Auengewässer waren primär eutroph!), die geringe Wassertiefe und das Vorhandensein entsprechender Vegetationsstrukturen unterstreichen die Eignung als Lebensraum, so daß wir annehmen, daß die Evolution der Art eng mit diesem Lebensraumtyp verknüpft war und die Seen (s. 5.7.3), die mit nur 10000 Jahren weitaus jünger sind, erst zusätzlich ins besiedelte Gewässertypenspektrum hinzukamen.

Auch aktuelle Funde zeigen einen gewissen Schwerpunkt in verbliebenen Auenresten: Mulde/ Sachsen-Anhalt (PETZOLD, 1994); Donau/Bayern (HESS, et al 1996), Rhein/Rheinland-Pfalz (KITT \& WOLF, 1995) und eigene unpublizierte Untersuchungsergebnisse in Saar-, Mosel- und Primstal sowie an der unteren Oder (1997) und am Pripjat in Weißrußland (1995). Dort gelang u. a. ein Exuviennachweis an einem sehr langsam fließenden Nebenarm.

Bei den 4 Exuvien-Funden von VernaUX (1972) handelt es sich nach MARTENS (1982) und JAQUEMIN u. a. (1985) um Fließgewässer.

\subsubsection{Epitheca bimaculata in pleistozänen Seenlandschaften}

Die letzte Inlandvereisung hinterließ eine enorme Anzahl unterschiedlich großer Standgewässer im Norden vor allem im Baltikum, in Polen, Brandenburg, Mecklenburg und SchleswigHolstein sowie im Süden im Alpenvorland. Als Habitat für Epitheca waren die größeren und tieferen Seen - einst oligotroph - vermutlich ungeeignet. Es verblieben jedoch noch Tausende primär meso- oder eutrophe Kleinseen als potentielle Brutgewässer, die womöglich nach der Laubwaldausbreitung einst flächendeckend besiedelt wurden. Vermutlich nur Reste dieser Vorkommen wurden in der vorliegenden Untersuchung in der Uckermark nachgewiesen.

\subsection{Anthropogene Schädigung von Epitheca-Populationen}

Anhand der vorgestellten weitgefächerten neuen Erkenntnisse über die Art sind auch Aussagen über die Auswirkung menschlicher Landschaftsveränderung und damit die Gefährdungsursachen für diese „Rote-Liste-Art" möglich. Zu erwähnen sind:

- Beseitigung von submersen und emersen Vegetationsstrukturen durch Hypertrophierung, Besatz pflanzenvernichtender Fischarten oder mechanische Einflüsse,

- Aufforstung mit Nadelbaumarten und Beseitigung von ufernahen Gehölzen (betrifft die seenreichen Jungpleistozänlandschaften),

- Trockenlegung von Flußauen mit ihren zahlreichen Stillgewässern und Rodung der Auenwälder (betrifft alle Flußsysteme der planaren bis kollinen Stufe). 


\section{Literatur}

ARGE IFÖNA/ÖKOTOP 1991: Schutz-, Pflege- und Entwicklungsplanung für das Saar-Altwasser bei Beckingen. - Unveröff. Gutachten im Auftrag des Ministers für Umwelt, Saarbrücken.

ASKEW, R. R. 1988: The dragonflies of Europe. - Harley Books, Colchester: 291 S.

BAUER, J. 1978: Libellenbeobachtungen im westlichen Allgäu. - Mitt. Arb.gem. Naturschutz Wangen im Allgäu 1: 23-33.

BELLMANN. H. 1987: Libellen: beobachten, bestimmen. - Neumann-Neudamm, Melsungen.

BORCHERDING, J.; BARTHOLD, K. \& BECKER, J. 1998: Entwicklungsnachweis für Brachytron pratense, Epitheca bimaculata, Libellula fulva und L. quadrimaculata (Odonata) in der Stopfenreuther Donauaue (Niederösterreich). - Lauterbornia 33: 13- 18.

BURCKHARDT, T. 1993: Durchführung und Überwachung einer ökologiegerechten Fischerei und Untersuchungen zur Ichthyofauna im Biosphärenreservat Schorfheide-Chorin. - Unveröff. Gutachten, LAGS Eberswalde.

COPPA, G. 1990: Élements cartographiques et ecologiques sur les odonates de Champagne-Ardenne. Publications scientifiques du Pavillion Saint-Charles. Association de Gestion de l'Unité de Recherche sur la Nature. Troyes: $105 \mathrm{~S}$.

COPPA, G. 1991: Notes sur l'émergence d'Epitheca bimaculata (CHARPENTIER)(Odonata: Corduliidae). Martinia 7 (1): 7-16.

CORBET, O. 1983: A biology of dragonflies. - Faringdon (Classey), $247 \mathrm{~S}$.

Degrange, C. \& Seassau, M.-D. 1974: Odonates Corduliidae de Savoie et du Dauphiné. - Trav. Lab. Hydrobiol. Grenoble 64/65: 289-308.

DOMMANGET, J.-L. 1994: Atlas préliminaire des odonates de France. Etat d'avancement au 31/12/93. Collion patrimoines naturelles, série patrimoine génétique 16: $92 \mathrm{~S}$.

DONATH, H. 1987: Vorschlag für ein Libellen-Indikatorsystem auf ökologischer Grundlage am Beispiel der Odonatenfauna der Niederlausitz. - Entomol. Nachr. u. Ber. 31 (5): 213-217.

DREYER, H. 1986: Die Libellen. - Gerstenberg-Verlag. Hildesheim.

Dufour, C. 1978: Etude faunistique des Odonates de Suisse Romande. - Conservation de la Faune et Section de Protection de la Nature et des Sites du Canton de Vaud, 146 S.

Duval, B. \& Pratz, J.-L. 1988: Présence d' Epitheca bimaculata en Forêt d'Orléans. - Bull. Ann. Nat. orl. Loire moy. 7: 89-93.

FRÖHLICH, H. 1900: Über das Vorkommen des E. bimaculata CHARP. - Entomol. Nachrichten 24: 379-382.

GfUG (Gesellschaft für Umweltschutz und Gewässerökologie) 1995: Fischereibiologische Untersuchungen der Saar-Auegewässer Schwemlingen, Niedmündung, Beckingen, Rehlingen und Ökosee Dillingen. - Gutachten im Auftrag der Naturlandstiftung Saar. Beckingen.

HEIDEMANN, H. \& SEIDENBUSCH, R. 1993: Die Libellenlarven Deutschlands und Frankreichs. Handbuch für Exuviensammler. - Keltern, Bauer-Verlag.

HESS, M.; HECKES, U. \& SCHÖN, M. 1996: Epitheca bimaculata (CHARPENTIER) in Bayern (Anisoptera: Corduliidae). - Libellula 15 (1/2): 27-44.

HEYER, E. 1962: Das Klima des Landes Brandenburg. - Abh. d. meteorol. u. hydrolog. Dienstes der DDR 64: 5-61.

JACOB, U. 1969: Untersuchungen zu den Beziehungen zwischen Ökologie und Verbreitung heimischer Libellen. - Faun. Abh. Staatl. Mus. Tierk. Dresden 2: 197-239.

JACQUEMIN, G.; Boudot, J.-P.; GouTET, P. \& SCHWAAB, F. 1985: Presence d'Epitheca bimaculta CHARP. en Lorraine (Odonata, Corduliidae). - Bulletin de la Société d'histoire naturelle de la Moselle, Quarante quatrième cahier: 229-242.

JAHN, P. 1982: Liste der Libellenarten (Odonata) von Berlin (West) mit Kennzeichnung der ausgestorbenen und gefährdeten Arten (Rote Liste). - Landschaftsentwicklung und Umweltforschung Nr. 11, 297-310.

JoHnSON, D. M. \& MOORE, R. D. 1991: Fish-mediated life-history strategies in the dragonfly Epitheca cynosura. - J. North Am. Benthol. Soc. 10 (3): 271-279.

JURZITZA, G. 1965: Die Eiablage der Zweiflecklibelle. - Natur 73: 304-306. 
KITT, M. \& WOLF, E. 1995: Der Zweifleck - Epitheca bimaculata (CHARPENTIER, 1825) in der südpfälzischen Rheinniederung. - Fauna Flora Rheinland-Pfalz 7 (4): 1077-1079.

LAWA (Länderarbeitsgemeinschaft Wasser) 1999: Gewässerbewertung - Stehende Gewässer. Vorläufige Richtlinie für eine Erstbewertung von natürlich entstandenen Seen nach trophischen Kriterien, 1998. Kulturbuch-Verl. Berlin.

L.E.G.U.A.N. 1993: Libellenkartierung im Rahmen der UVS A20, Rostock bis Bundesgrenze. - Kartierung im Auftrag von Geologie und Umwelt GFE, Schwerin.

LE ROI, O. 1915: Die Odonaten der Rheinprovinz. - Verh. Naturh. Ver. Preuss. Rheinl. 1915: 119-178.

LETT, J.-M. 1989: Presence d Epitheca bimaculata (CHARPENTIER, 1825) dans le departement du Loir-etcher (41) (Odonata, Anisoptera: Corduliidae). - Martinia 5 (2): 36.

LOHMANN, H. 1980: Faunenliste der Libellen der Bundesrepublik Deutschland und Westberlins. Societas internationalis odonatologica, Rap. Comm. 1: 1-34.

MARTENS, K. 1982: New localities for E. bimaculata CHARP. with a review of its status in western Europe. - Notul. odonatol. 1 (10): 157-159.

MAUERSBERGER, R. \& MAUERSBERGER, H. 1992: Odonatologischer Jahresbericht aus dem Biosphärenreservat „Schorfheide-Chorin“ für 1992. - Libellula 11 (3/4): 155-164.

MAUERSBERGER, R. \& WAGNER, S. 1990: Zur Libellenfauna dreier Naturschutzgebiete im Bezirk Rostock. - Naturschutzarbeit in Mecklenburg-Vorpommern 33 (1): 23- 29.

MAUERSBERGER, R. 1993: Gewässerökologisch-faunistische Studien zur Libellenbesiedlung der Schorfheide nördlich Berlins. - Arch. f. Nat.-Lands. 32: 85-111.

MAUERSBERGER, H.\&MAUERSBERgER, R. 1996: Die Seen des Biosphärenreservates Schorfheide-Chorin - eine ökologische Studie. Untersuchungen zur Struktur, Trophie, Hydrologie, Entwicklung, Vegetation und Libellenfauna. - Unveröff. Diss. Ernst-Moritz-Arndt Universität Greifswald - 741 S.: 318 Abb., 102 Tab.

MeYnen, E.; SChmidthüsen, J.; Gellert, J.; NeEF, E.; MÜller-MINY, H. \& SChultze, H. J. 1959: Handbuch der naturräumlichen Gliederung Deutschlands. 6. Lieferung.

MOTHES, G. 1965: Die Odonaten des Stechlinsees. - Limnologica (Berlin) 3 (3): 389-397.

MÜNCHBERG, P. 1932: Beiträge zur Kenntnis der Biologie der Libellenunterfamilie der Corduliinae. Intern. Rev. Ges. Hydrobiol. 27: 265-302.

NIEHUIS, M. 1984: Verbreitung und Vorkommen der Libellen (Insecta: Odonata) im Regierungsbezirk Rheinhessen-Pfalz und im Nahetal. - Naturschutz u. Ornithologie in Rheinland-Pfalz 3 (4): 536-607.

PETzOLD, F. 1994: Bemerkenswerte Libellenfunde in der Muldeniederung bei Dessau. - Libellula 13 (1/2): 33-46.

REDER, G. 1992: Erste Fortpflanzungsnachweise des Zweiflecks - Epitheca bimaculata (CHARPENTIER, 1825) - in Rheinland-Pfalz (Insecta: Odonata). - Fauna und Flora in Rheinland-Pfalz 6 (4): 1152-1156.

ROBERT, P.-A. 1959: Die Libellen (Odonaten). - Verlag Kümmerly \& Frey, Bern, 404 S.

SANDHALL, A. 1987: Trollsländor i Europa. - Interpublishing, Stockholm, $251 \mathrm{~S}$.

SCHIEMENZ, H. 1953: Die Libellen unsere Heimat. - Jena, Urania-Verlag. 154 S.

SCHMIDT, E. 1975: Die Libellenfauna des Lübecker Raumes. - Ber. Ver. H. Naturhist. Mus. Lübeck 13/14: $25-43$.

SCHMIDT, E. 1988: Schluchtsee und Heider Bergsee im Braunkohlenrekultivierungsgebiet der Ville bei Köln: Sekundärbiotope vom Charakter der Flußaltarme im Konflikt mit Freizeitnutzungen. - Verh. Westd. Entom. Tag 1988: 103-116.

SCHMIDT, E. 1989: Libellen als Bioindikatoren für den praktischen Naturschutz: Prinzipien der Geländearbeit und ökologische Analyse und ihre theoretische Grundlegung im Konzept der ökologischen Nische. - Schriftenr. f. Landschaftspfl. u. Natursch. 29: 281-289.

SCHORR, M. 1990: Grundlagen zu einem Artenhilfsprogramm Libellen der Bundesrepublik Deutschland Ursus Scient. Publishers, Bilthoven.

SONEHARA, 1. 1967: On the life history of dragonflies of the genus Epitheca with special reference to $E$. bimaculata sibirica SÉLYS in Mt. Yatsugatake. - Tombo, Tokyo 10 (1-4): 2-24.

STERnBERG, K. 1990: Autökologie von sechs Libellenarten der Moore und Hochmoore des Schwarzwaldes und Ursachen ihrer Moorbindung. - Diss. Albert-Ludwigs-Univ. Freiburg i. Br.; 431 S. 
ST. QUENTIN, D. 1960: Die Odonatenfauna Europas, ihre Zusammensetzung und Herkunft. - Zool. Jahrb. Syst. 87 (4-5): 301-315.

Succow, M. 1988: Landschaftsökologische Moorkunde. - Gustav Fischer Verl., Jena.

TGL 27885/01 1982: Nutzung und Schutz der Gewässer - Stehende Binnengewässer - Klassifizierung. -

Ministerium für Umweltschutz und Wasserwirtschaft der DDR, Berlin.

TrockUR, B. 1989: Erstnachweis des Zweiflecks (Epitheca bimaculata CHARPENTIER 1825, Insecta: Odonata), einer bundesweit vom Aussterben bedrohten Falkenlibelle, für das Saarland. - Faunistischfloristische Notizen aus dem Saarland 1/2: 1-16.

TROCKUR, B. 1990: Aktuelles, bodenständiges Vorkommen von Epitheca bimaculata (CHARPENTIER, 1825) im Saarland (Anisoptera: Corduliidae). - Libellula 9 (3/4): 93-103.

TROCKUR, B. 1993: Erste Ergebnisse von Untersuchungen zum Epitheca-Vorkommen im Saarland (Anisoptera: Corduliidae). - Libellula 12 (3/4): 139-151.

TROCKUR, B. 1997: Bemerkenswerte Libellenfunde im Kiesweihergebiet bei Remerschen: Wiederfund von Epitheca bimaculata und Erstnachweis von Anax parthenope für Luxemburg (Insecta, Odonata). Bull. soc. nat. luxemb. 98: 105-112.

TROCKUR, B. \& MAUERSBERGER, R. in prep.: Zur Verbreitung von Epitheca bimaculata in Deutschland. VerneAUX, J, 1972: Faune dulçaquicole de Franche-Comté: le Bassin du Doubs. 5/Les Odonates. - Ann. scientif. Univ. Besançon, Zool., Physiol. et Biol. anim., 3ème série (8): 15-20.

VINCENT, G.; BOUDET, J.-P.; JACQUEMIN, G.; GOUTET, P. \& SCHWAB, F. 1987: Epitheca bimaculata (CHARPENTIER, 1825) dans l'est de la France: Rare, ou discrete et meconnue? (Odonata Anisoptera: Corduliidae). - Martinia 6: 3-13.

Anschrift der Verfasser:

BERND TROCKUR

Schulstraße 4

D - 66636 Tholey-Scheuern

Deutschland

\author{
Dr. RÜDIGER MAUERSBERGER \\ Waldstraße 4 \\ D - 16278 Steinhöfel \\ Deutschland
}

\section{Besprechungen}

RÖSER, B.: Grundlagen des Biotop- und Artenschutzes. - 2. Aufl. - Landsberg/Lech: ecomed, 1995. 176 S.: 46 Abb. - ISBN 3-609-65930-0

Naturschutz war Anfang der 70er Jahre für viele Bundesbürger kein Thema, mit dem konkrete Inhalte verbunden waren, es sei denn, man dachte an die zentralafrikanischen und nordamerikanischen Nationalparks. So reduzierte sich auch Artenschutz auf die Erhaltung der letzten Großsäugetiere, wie Wale, Tiger, Naßhörner, Elefanten. „Dank der Arbeit von Umwelt- und Naturschutzverbänden, von Funk, Fernsehen und Presse ist Naturschutz heute in aller Munde, doch sind die erforderlichen Maßnahmen zum Erhalt des Tier- und Pflanzenartenspektrums und ihrer Lebensräume erst in spärlichen Ansetzen in die Wege geleitet $^{65}$. Diese Aussage des Verfassers aus dem Jahre 1990 hat leider wenig an Aktualität eingebüßt. So erschien auch 1995 diese zweite unveränderte Auflage des Werkes.

Die Grundlagen des Biotop- und Artenschutzes gliedern sich in die Abschnitte:

1. Einführung, 2. Ausmaß der Arten- und Lebensraumgefährdung, 3. Naturschutz und Landschaftspflege (Argumente), 4. Ursachen der Arten- und Biotopvernichtung, 5. Naturschutz im Lichte der öffentlichen Meinung, 6. Naturschutz und Landschaftspflege (alte und neue Strategien), 7. Ausblick, 8. Literaturverzeichnis, 9. Anhang, 10. Stichwortverzeichnis. 